\title{
New formulations of the Hop-Constrained Minimum Spanning Tree problem via Miller-Tucker-Zemlin constraints
}

\author{
İbrahim Akgün*, Barbaros Ç. Tansel \\ Department of Industrial Engineering, Bilkent University, Bilkent 06800, Ankara, Turkey
}

\section{A R T I C L E I N F O}

\section{Article history:}

Received 5 April 2010

Accepted 28 January 2011

Available online 3 February 2011

\section{Keywords:}

Graph theory

Integer programming

Spanning trees

Hop constraints

Miller-Tucker-Zemlin constraints

\begin{abstract}
A B S T R A C T
Given an undirected network with positive edge costs and a natural number $p$, the Hop-Constrained Minimum Spanning Tree problem (HMST) is the problem of finding a spanning tree with minimum total cost such that each path starting from a specified root node has no more than $p$ hops (edges). In this paper, we develop new formulations for HMST. The formulations are based on Miller-Tucker-Zemlin (MTZ) subtour elimination constraints, MTZ-based liftings in the literature offered for HMST, and a new set of topologyenforcing constraints. We also compare the proposed models with the MTZ-based models in the literature with respect to linear programming relaxation bounds and solution times. The results indicate that the new models give considerably better bounds and solution times than their counterparts in the literature and that the new set of constraints is competitive with liftings to MTZ constraints, some of which are based on well-known, strong liftings of Desrochers and Laporte (1991).
\end{abstract}

(c) 2011 Elsevier B.V. All rights reserved.

\section{Introduction}

Minimum spanning tree problems arise quite naturally in transportation and communication network design when it is necessary to provide a minimum-cost connectivity among a number of geographically dispersed locations or system components. Various examples of minimum-cost tree networks are given by Ahuja et al. (1993) from network design in transportation, telecommunication, data storage, and cluster analysis. We consider in this paper the Hop Constrained Minimum Spanning Tree (HMST) problem, studied earlier by Gouveia (1995), which involves designing a minimum-cost spanning tree such that each path in the tree from a specified root node to every other node has no more than $p$ hops (edges).

Gouveia (1995) has given several formulations of the HMST problem based on the well-known Miller-Tucker-Zemlin (MTZ) subtour elimination constraints (Miller et al., 1960) and has provided various liftings to MTZ constraints, some of which are based on the strong liftings of Desrochers and Laporte (1991). Linear programming (LP) bounds and Lagrangean relaxation bounds based on subgradient optimization have been offered. Reported computational results in that paper on complete graphs with up to 40 nodes indicate that lower bounds resulting from LP or Lagrangean relaxations are weak.

In this paper, we propose new formulations of the HMST problem to improve LP bounds and solution times. We propose new

\footnotetext{
* Corresponding author. Tel.: +90 312290 1960; fax: +90 3122664054 .

E-mail address: iakgun@bilkent.edu.tr (İ. Akgün).
}

sets of constraints to strengthen the MTZ subtour elimination constraints as well as hop-related topology-enforcing constraints. Our computational tests indicate that the formulations we propose give considerably better LP bounds and solution times than previous formulations studied earlier by Gouveia (1995).

Hop constraints arise in designing local access networks where a central processing unit (computer) communicates with many terminals at geographically dispersed locations that are connected to it via multidrop transmission lines. A tree structure leads to a better utilization of line capacities due to shared usage. Such networks generally enjoy low message traffic. Congestion is either rare or nonexistent. While transmission time in any one link is negligible due to low traffic, traversal of many links during transmission leads to non-negligible delays that are kept under control by restricting the number of hops traversed during transmission. Hop constraints are also relevant for networks with links that are prone to possible failure. Requiring that all messages be successfully transmitted with a certain threshold probability can be expressed as a hop constraint assuming that link failures are independent identically distributed random variables. Woolston and Albin (1988) show, for example, that spanning tree designs with an upper bound on the number of hops perform better with respect to successful message transmission than those without such bounds. LeBlanc et al. (1999), Balakrishnan and Altinkemer (1992), and Gouveia et al. (2003) discuss applications of hop constraints in more general network design problems. Dahl (1998) also defines applications in transportation, statistics, and plant location for the case with at most two hops per path.

To define the HMST problem, let $G=(V, E)$ be an undirected connected network with node set $V=\{r, 1, \ldots, n\}$, edge set $E$, and 
positive edge costs $c_{e}(e \in E)$. Node $r$ represents the central processing unit and is referred to as the root node.A spanning tree of $G$ is a connected sub-graph of $G$ that has no cycles and spans all nodes. Given a positive integer $p$, a spanning tree is a hop-constrained spanning tree or a feasible tree if the unique path from the root node to any other node has no more than $p$ hops. The HMST problem is the problem of finding a feasible tree whose total cost is minimum. If $p \geqslant n$, all spanning trees are feasible and hop constraints can be ignored. If $p=1$, either there is no feasible tree or there is exactly one feasible tree which is the star tree with the root node at its center. We assume $2 \leqslant p \leqslant n-1$ from now on.

While the unconstrained minimum cost spanning tree problem is solvable in low order polynomial time by the algorithms of Kruskal (1956) and Prim (1957), the HMST problem is NP-Hard (Gouveia, 1995; Dahl, 1998). Manyem and Stallmann (1996) show that the HMST problem is not in the class APX (the class of problems for which it is possible to have a polynomial time heuristic with a guaranteed approximation bound). Dahl (1998) studies the HMST problem for $p=2$ and compares the polyhedra of the models with directed and undirected arcs. Alfandari and Paschos (1999) show that the case with $p=2$ cannot be approximated by polynomial time approximation schemes unless $P=$ NP.

A version of the HMST problem that limits the number of nodes rather than edges on each path is introduced at a rudimentary level by Gavish (1985). Gouveia (1995) formulates the HMST problem using the MTZ subtour elimination constraints and studies the LP and Lagrangean relaxation bounds. An alternative formulation is offered by Gouveia (1996) based on directed or undirected multicommodity flows (MCF). Even though the LP bounds of MCF formulations are considerably better than those of MTZ formulations, MCF formulations lead to very large integer programming models whose LP relaxations require excessive solution times and core storage as the network size and $p$ get larger (Dahl et al., 2006). Additional improvements are obtained for small values of $p$ using a hop-indexed formulation (Gouveia, 1998). MTZ formulations are much more compact in the numbers of variables and constraints than MCF based formulations. There are problem instances, for example, where an MTZ formulation finds an integer feasible solution in seconds whereas an MCF formulation cannot even solve the LP relaxation in days (Dahl et al., 2006). Computational results also indicate that it may take a considerable amount of time to arrive at an optimal integer solution even if the gap between the LP and integer optimal values is quite small. Due to significant differences in model sizes and in solution times between MTZ and MCF formulations, we focus in this study on the former.

Other related works on the HMST problem include lower bounding schemes based on Lagrangean relaxation (Gouveia, 1998). Reported computational results indicate that Lagrangean based bounds are much better than LP bounds of the MCF formulation for small values of $p$ and that it is very time-consuming to obtain bounds that are close to the theoretically possible best bounds. To overcome this inefficiency, Gouveia and Requejo (2001) give a Lagrangean-based bounding scheme using a hopindexed formulation. Even though this approach gives better bounds than the aforementioned ones, it is dependent on the value of $p$ and performs poorly as $p$ increases.

Dahl et al. (2004) introduce a new formulation for the HMST problem using only natural design variables and an exponential number of constraints composed of the so-called jump inequalities that are shown to be facet-defining. Their proposed formulation uses fewer variables but has weaker LP bounds than MCF formulations. Due to the exponential number of constraints, the authors propose a Lagrangean-based bounding scheme. Computational results indicate that the LP bounds are better as $p$ increases than those reported in previous studies. Dahl et al. (2006) summarize the aforementioned approaches. Kerivin and Mahjoub (2005) give a survey of several network design problems with hop constraints and methods to solve them. More general telecommunication network design problems are also considered using MCF formulations (Balakrishnan and Altinkemer, 1992) or hop-indexed formulations (Pirkul and Soni, 2003; Gouveia et al., 2003).

The models described in the aforementioned papers view the HMST problem as defined in the original graph (although some of these models view the underlying hop-constrained shortest path problem as defined in an appropriate layered graph). Gouveia et al. (2010) propose a modeling approach that views the whole problem as defined in a single layered graph. They show that the HMST problem is equivalent to a Steiner tree problem (Hwang et al., 1992; Maculan, 1987) in an adequate layered graph. They prove that the LP bounds obtained by the directed cut Steiner tree formulation on a layered graph with an exponential number of constraints is better than the best known ones in the literature. Computational results with a branch-and-cut algorithm show that the proposed method is significantly better than previously known methods.

Gouveia et al. (2008) study a distance-constrained version of the HMST problem where edges have different transmission delays and the bound $p$ on the number of hops is replaced by a bound on the sum of edge delays along any path from the root node. The authors present a column generation scheme, a Lagrangian relaxation procedure combined with subgradient optimization, and a shortest path (compact) reformulation which views the underlying subproblem as defined on an extended layered graph. Computational results show that the layered-graph path formulation is better than the other two approaches.

A closely related problem is the Diameter-Constrained MST problem where the aim is to find a minimum-cost spanning tree such that the unique path between any pair of nodes has no more than $p$ hops. Some notable contributions on this problem are Achuthan et al. (1994), Gouveia and Magnanti (2003), Gruber and Raidl (2005), Santos et al. (2004), and Gouveia et al. (2010).

Our focus in the paper is on new MTZ based formulations that give better LP bounds and better solution times than the previous MTZ based formulations with or without liftings. The remainder of the paper is organized as follows. Section 2 reviews the existing MTZ based models of the HMST problem studied in Gouveia (1995). Section 3 gives our proposed formulations. Section 4 gives a description of the test problems. Section 5 compares new and existing models on the basis of LP bounds. Section 6 compares existing and new models on the basis of solution times. Section 7 concludes the paper.

\section{Existing MTZ based formulations of the HMST problem}

In this section, we give the formulations of the HMST problem studied by Gouveia (1995). We find it convenient to view the constraints of the minimum spanning tree problems with additional requirements on the structure of the tree in two subsets, tree-defining and topology-enforcing. This distinction is made earlier by Akgun and Tansel (2010) in the context of the Minimum-Degree Constrained Minimum Spanning Tree problem and provides a more structured way of assessing effects of different sets of constraints on LP bounds and solution times. The tree-defining portion of the constraints consists of those that ensure that a spanning tree is obtained from a given edge weighted graph. The topology-enforcing portion of the constraints consists of those that ensure that the resulting spanning tree satisfies the additional structural requirements imposed on the topology of the tree. For example, hop constraints or degree constraints belong to the topology-enforcing set. Tree-defining constraints do not depend on the particular topological requirements of the problem under study and thus can be viewed as a core part of any spanning tree problem regardless of 
additional requirements that may be present on the topology of the tree. The topology enforcing set is problem-specific and, in the context of the HMST problem, it refers to the set of constraints that are related to the hop requirement. Supplemental constraints may also be present in the formulation to improve LP bounds or CPU times. Supplemental constraints may be included in the tree-defining or topology-enforcing sets as appropriate.

Tree-defining constraints may be based on packing, cut-sets, single/multi-commodity flows, or MTZ subtour elimination constraints (Magnanti and Wolsey, 1995). MTZ constraints are attractive due to their compactness, but they are well-known for producing weak LP bounds. Orman and Williams (1999) compare the strengths of several formulations of the well known Travelling Salesman Problem (TSP), including the ones with MTZ and flow constraints, on the basis of their LP bounds. The reader is referred to Nemhauser and Wolsey (1988), Gutin and punnen (2002), and Applegate et al. (2007) for additional information on the TSP. Orman and Williams (1999) find out that the LP polytope of the TSP resulting from MTZ constraints contains some of the seven existing formulations of the TSP. This has led to various studies to augment the MTZ constraints and strengthen the LP bounds of the TSP formulation (Desrochers and Laporte, 1991; Gouveia and Pires, 1999; Sherali and Driscoll, 2002). The formulations and liftings proposed in the context of the TSP can also be used in other problems where subtours are not allowed. Gouveia (1995) uses the MTZ constraints to formulate the HMST problem and offers liftings of his own as well as liftings adapted from Desrochers and Laporte (1991).

The HMST problem is defined on a directed network $G^{\prime}=(V, A)$ obtained from the undirected graph $G=(V, E)$ by replacing each undirected edge $\{i, j\} \in E$ by two directed arcs $(i, j)$ and $(j, i)$ with symmetric costs $c_{i j}=c_{j i}$. A feasible solution to the HMST problem defines a subgraph of $G^{\prime}=(V, A)$ that spans all nodes in $V$, is connected, has no cycles, and has exactly one incoming arc at each node except the root node. The root node has no incoming arc. There is no restriction on the number of outgoing arcs at any node. Such a subgraph is referred to as an arborescence (Ahuja et al., 1993). An arborescence is a spanning tree if arc directions are ignored. Each path in an arboresence originating at the root node is a directed path consisting of arcs that have the same direction (away from the root node).

Two sets of decision variables are used in the formulation of the HMST problem based on the MTZ constraints: (1) binary design variables $x_{i j}$ that take on the value of 1 if $\operatorname{arc}(i, j)$ is in the design and 0 , otherwise, and (2) non-negative node-labeling variables $u_{i}$ that increase monotonically along nodes of any path originating at the root node.

We summarize in what follows the models and liftings offered by Gouveia (1995). We use the acronym $\mathbf{a} / \mathbf{b}$ to label the models where "a" refers the tree-defining part and "b" refers to the topologyenforcing part. For the models of Gouveia (1995), "a" may stand for "Basic MTZ Constraints (BMTZ)" while "b" may stand for "Basic Topology Enforcing Constraints (BTEF)" or "Extension $i$ of the BTEF constraints (EiBTEF) for $\boldsymbol{i}=1, \ldots$, 8." Accordingly, BMTZ/BTEF which we refer to as the Basic Model, represents the model with Basic MTZ Constraints and BTEF Constraints while BMTZ/EiBTEF represents the model with Basic MTZ Constraints and Extension $\boldsymbol{i}$ of BTEF Constraints. We refer to the model BMTZ/EiBTEF as the Extension $i$ of the Basic Model.

The first MTZ based model of the HMST problem that we give below is the Basic Model or BMTZ/BTEF. The basic MTZ constraints in BMTZ/BTEF are the constraints (1)-(5) and the basic topology enforcing constraints are the hop constraints (6). Constraints (7) and (8) are nonnegativity and binary restrictions and are not viewed as part of tree-defining or topology-enforcing sets.
BMTZ/BTEF: Basic Model

Basic MTZ Constraints/Basic Topology Enforcing Constraints

$$
\begin{array}{ll}
z^{*}=\min _{x, u} & \sum_{(i, j) \in A} c_{i j} x_{i j} \\
\text { s.t. } & \sum_{i} x_{i j}=1 \quad j \in(V-r), \\
& u_{i}-u_{j}+n x_{i j} \leqslant n-1 \quad(i, j) \in A, j \neq r, \\
& u_{i}=0 \quad i=r, \\
& u_{i} \geqslant 1 \quad i \in(V-r), \\
& u_{i} \leqslant p \quad i \in(V-r), \\
& u_{i} \geqslant 0 \quad \forall i, \\
& x_{i j} \in\{0,1\} \quad i \in V, j \in(V-i-r) .
\end{array}
$$

A few remarks on the MTZ constraints are in order. The MTZ constraints are proposed initially by Miller et al. (1960) in the context of the TSP and consists of the constraints (3)-(5) and the additional constraints $u_{i} \leqslant n-1$ for $i \neq r$.The node labels $u_{i}$ in the context of the TSP formulation refers to the rank order of node $i$ in any feasible TSP tour with root node $r$ receiving the label $u_{r}=0$ and with node $i$ receiving the label $u_{i}=k$ if it is the $k$ th node in the tour. Note that (3) requires $u_{j}=u_{i}+1$ whenever node $j$ is visited immediately after node $i$ (that is, whenever $x_{i j}=1$ ). The whole range of node labels is used and node labels are uniquely assigned in the context of the TSP. The uniqueness of node labels is not required and the whole range of node labels need not be used in the context of the HMST problem. This permits to have more freedom of choice in the assignment of labels, a fact we exploit in the next section to improve our formulations. For example, a feasible solution where the same label is not assigned to all nodes with the same number of hops from the root is possible. Observe that constraints (3) prevent sub-tours by assigning labels in such a way that each directed arc included in the arborescence is directed from a node with a lower label into a node with a higher label. This ensures that node labels on any path form a monotonically increasing sequence, preventing thereby formation of cycles. Constraint (4) assigns a label of 0 to the root node. Constraints (5) and (6) define lower and upper bounds on labels that can be assigned to nonroot nodes, respectively. Specifically, in the assignment of labels to nodes, there are three possible cases for an edge $\{i, j\}$ : either $x_{i j}=1$, or $x_{j i}=1$, or both $x_{i j}$ and $x_{j i}=0$. If $x_{i j}=1$, then $u_{j} \geqslant u_{i}+1$. Similarly, if $x_{j i}=1$, then $u_{i} \geqslant u_{j}+1$. If both $x_{i j}=0$ and $x_{j i}=0$, then $u_{i}-u_{j} \leqslant n-1$ and $u_{j}-u_{i} \leqslant n-1$. In this respect, any assignment of labels satisfying the aforementioned conditions gives a feasible solution.

Note that the upper bounds on the labels defined by constraints (6) eliminate all paths with more than parcs. Constraints (5) are actually not required for the model to work properly and not used in the models studied in Gouveia (1995) as those constraints are not a part of original MTZ constraints (Miller et al., 1960) but added later. We prefer to include them to be in compliance with the recent approach in the literature and the proposed models. Constraints (2) require that the number of incoming arcs to any non-root node be equal to 1 , which, together with constraints (3), establish that the resulting tree is an arboresence. Objective function (1) minimizes the total cost of the arcs in the solution.

In any feasible solution to BMTZ/BTEF, constraints (6) ensure that the node labels satisfy the relation $u_{i}-u_{j} \leqslant p-1$ for any pair of nodes $i$ and $j$. Thus, constraints (3) can be modified to obtain a new set of topology-enforcing constraints, namely, constraints (9), to replace constraints (3) and (6). This gives the following extended formulation which we refer to as Extension 1 of the Basic Model (abbreviated as BMTZ/E1BTEF). Miller et al. (1960) used constraints (9) in the context of a vehicle routing problem to restrict the number of nodes visited in each tour. 
BMTZ/E1BTEF: Extension 1 of the Basic Model

(1), (2), (4), (5), (7), (8), and

$u_{i}-u_{j}+p x_{i j} \leqslant p-1 \quad(i, j) \in A, j \neq r$.

Following Desrochers and Laporte (1991), constraints (9) can be lifted to obtain constraints (10) that require the values of variables $u_{i}$ and $u_{j}$ to differ exactly by 1 if edge $\{i, j\}$ is in the solution, i.e., whenever $x_{i j}=1$ or $x_{j i}=1$. Formulation BMTZ/E2BTEF, Extension 2 of the Basic Model, is obtained by replacing constraints (9) with constraints (10).

BMTZ/E2BTEF: Extension 2 of the Basic Model

(1), (2), (4), (5), (7), (8), and

$(p-2) x_{j i}+u_{i}-u_{j}+p x_{i j} \leqslant p-1 \quad(i, j) \in A, j \neq r$.

A new set of liftings to constraints (9) can be obtained by providing additional information on the values of the variables $u_{i}$ and $u_{j}$. Note that $x_{i j}=0$ when $\sum_{k \neq i, r} x_{k j}=1$ due to constraints (2) and hence node $j$ cannot be adjacent to node $r$ when this occurs. Thus, the difference between the values of $u_{i}$ and $u_{j}$ is at most $p-2$. This result and the fact that $x_{i j}+x_{j i} \leqslant 1$ can be combined to obtain liftings (11) valid for $p \geqslant 3$. Formulation BMTZ/E3BTEF, Extension 3 of the Basic Model, is obtained by replacing constraints (9) with constraints (11) in the topology-enforcing set.

BMTZ/E3BTEF: Extension 3 of the Basic Model

(1), (2), (4), (5), (7), (8), and

$\sum_{k=1, k \neq i} x_{k j}+(p-3) x_{j i}+u_{i}-u_{j}+p x_{i j} \leqslant p-1 \quad(i, j)$

$\in A, j \neq r, p \geqslant 3$.

The model BMTZ/E4BTEF,Extension 4 of the Basic Model, enables us to evaluate the combined effects of liftings (10) and (11) in the topology enforcing part and is defined as follows.

BMTZ/E4BTEF: Extension 4 of the Basic Model

(1), (2), (4), (5), (7), (8), (10), and (11).

The following liftings for constraints (5) and (6) strengthen the LP bounds.

$u_{j} \leqslant p-(p-1) x_{i j} \quad i=r, j \in(V-r)$,

$u_{j} \geqslant 2-x_{i j} \quad i=r, j \in(V-r)$.

Constraints (12) and (13) require that the value of $u_{j}$ be equal to 1 if node $j$ is incident to the root node and greater than 2 if not. The following four models are proposed by Gouveia (1995) to see the effects of liftings (12) and (13).

BMTZ/E5BTEF: Extension 5 of the Basic Model

(1), (2), (4), (5), (7), (8), (9), (12), and (13).

BMTZ/E6BTEF: Extension 6 of the Basic Model

(1), (2), (4), (5), (7), (8), (10), (12), and (13).

BMTZ/E7BTEF: Extension 7 of the Basic Model

(1), (2), (4), (5), (7), (8), (11), (12), and (13).

BMTZ/E8BTEF: Extension 8 of the Basic Model

(1), (2), (4), (5), (7), (8), (10), (11), (12), and (13).

Notice that the model BMTZ/EiBTEF for $\boldsymbol{i}=\mathbf{1 , 2 , 3 , 4}$ and the model BMTZ/EjBTEF with $\boldsymbol{j}=\boldsymbol{i}+\mathbf{4}$ are exactly the same except for constraints (12) and (13). Thus, the models BMTZ/EjBTEF for $\boldsymbol{j}=\mathbf{5 , 6 , 7 , 8}$ allow us to assess the effects of constraints (12) and (13) over the corresponding models BMTZ/EiBTEF for $\boldsymbol{i}=\mathbf{1 , 2}$, 3,4 .

\section{New formulations for the HMST problem}

The basic topology-enforcing constraints (6) establish the hop requirement by imposing the upper bound $p$ on the node labels $u_{i}$. The extensions discussed in the previous section of the basic model are obtained by liftings that make adjustments on the values of the node labels. In this sense, only one topological aspect of the problem is exploited by existing formulations. Even though not directly stated in its definition, the HMST problem has additional exploitable aspects in the topological structure of feasible trees. In what follows, we first improve the basic topology-enforcing constraints by distinguishing leaf nodes (nodes of degree one) from central nodes (nodes of degree greater than one) in feasible trees and taking advantage of implied degree requirements as well as other structural implications that are valid for trees. Later, we give an improved version of the basic MTZ constraints. The Improved MTZ Constraints and the Improved Topology Enforcing Constraints will be referred to as "IMTZ" and "ITEF," respectively. We obtain the new formulations by combining basic or improved MTZ constraints with improved topology-enforcing constraints or extensions of improved topology-enforcing constraints that incorporate liftings. This produces ten new formulations of the HMST problem. The models are referred to as BMTZ/ITEF, IMTZ/ITEF, and IMTZ/EiITEF for $\boldsymbol{i}=\mathbf{1}, \ldots, \mathbf{8}$ in accordance with the naming convention adopted for the models of Gouveia (1995). Here, BMTZ/ITEF stands for the model with Basic MTZ Constraints and ITEF Constraints, IMTZ/ITEF stands for the model with Improved MTZ Constraints and ITEF Constraints, and IMTZ/EiITEF stands for the model with Improved MTZ Constraints and Extension $\boldsymbol{i}$ of Improved Topology Enforcing Constraints. The models IMTZ/ EiITEF for $\boldsymbol{i}=\mathbf{1}, \ldots, \mathbf{8}$ are obtained by incorporating the liftings (with necessary adaptations as necessary) that are discussed in the previous section and referred to as Extension $i$ of the model IMTZ/ITEF.

We first give the improved version of the topology-enforcing constraints.

\subsection{Improved Topology-Enforcing Constraints (ITEF)}

In a feasible solution to the HMST problem, a node is either a leaf node or a central node. Leaf-nodes have degrees of one while central nodes have degrees of more than one. If the root node is a leaf-node, it has one outgoing arc but no entering arcs. Any nonroot node that is a leaf- node has one incoming arc but no outgoing arcs. If the root node is a central node, it has two or more outgoing arcs but no incoming arc while a non-root node that is a central node has one incoming arc and at least one outgoing arc. Thus, the number of arcs incident to a node can be restricted as dependent on the type of the node. Moreover, whether or not an arc may exist between two nodes may be established as dependent on the type of the nodes. If both nodes are leaf nodes, then no arc may exist between them. Additionally, only a single arc in one direction can be permitted between any two nodes. We now give the improved topology-enforcing constraints based on the foregoing.

Let $w_{i c}$ and $w_{i l}$ be a pair of binary variables associated with node $i$ with $w_{i c}=1\left(w_{i l}=1\right)$ if node $i$ is a central (leaf) node and $w_{i c}=0$ $\left(w_{i l}=0\right)$ if not.

ITEF: Improved Topology-Enforcing Constraints

In addition to hop-enforcing constraint (6)

$w_{i c}+w_{i l}=1 \quad i \in V$,

$\sum_{j} x_{i j} \geqslant 1 \quad i=r$

$\sum_{j \neq r} x_{i j} \geqslant 1+w_{i c} \quad i=r$, 
$\sum_{j \neq r} x_{i j} \leqslant(n-1)-(n-2) w_{i l} \quad i=r$

$\sum_{j} x_{j i}+\sum_{j \neq r} x_{i j} \geqslant 1+w_{i c} \quad i \in(V-r)$,

$\sum_{j} x_{j i}+\sum_{j \neq r} x_{i j} \leqslant(n-1)-(n-2) w_{i l} \quad i \in(V-r)$,

$\sum_{j \neq r} x_{i j} \geqslant 1-w_{i l} \quad i \in(V-r)$,

$x_{i j} \leqslant w_{i c} \quad(i, j) \in A, i \neq r, j \neq r$,

$x_{i j}+w_{i l}+w_{j l} \leqslant 2 \quad(i, j) \in A, j \neq r$,

$x_{i j} \equiv 0 \quad(i, j) \in A, j=r$,

$x_{i j}+x_{j i} \leqslant 1 \quad(i, j) \in A, i<j$,

$\sum_{j \neq i} x_{i j}=n-1$,

$w_{i c}, w_{i l} \in\{0,1\} \quad i \in V$.

Constraints (15) require that each node be either a leaf node or a central node. Constraints (16)-(18) define lower and upper bounds on the number of outgoing arcs from the root node. Constraint (16) establishes that the number of outgoing arcs at the root node $r$ is at least one. This constraint is redundant but helps to improve solution times. Constraints (17) and (18) require that the number of outgoing arcs at the root node be exactly one if $r$ is a leaf node and be in the range $[2, n-1]$ if $r$ is a central node.

Constraints (19)-(21) set upper and lower limits on the degrees of non-root nodes. The left sides of (19) and (20) give the total number of arcs incident at each non-root node. The right sides take on different values depending on if the node is a central node or a leaf node. Constraints (19) and (20) jointly ensure that the total number of arcs incident at a non-root node is exacly 1 if it is a leaf node. This and constraints (2) imply that there is exactly 1 incoming arc and no outgoing arc at such a node. If a non-root node is a central node, however, constraints (19) and (20) ensure that the total number of arcs incident at that node is in the range $[2, n-1]$. This and constraints (2) imply that there is exactly 1 incoming arc and at least 1 outgoing arc at such a node. Constraints (21) require that the number of outgoing arcs at a non-root node is greater than $1(0)$ when the node is a central (leaf) node, which is something already implied by constraints (2), (19), and (20). Even though constraints (21) are implied by other constraints, they are included in the formulation because the computational studies that we have carried out indicate that their presence improves the solution times.

Constraints (22) require that a non-root node be a central node if there is an outgoing arc from it. Constraints (23) prevent arcs between pairs of leaf nodes. Constraints (24) do not allow any arcs incoming to the root node. Constraints (25) state that a pair of arcs of opposite directions between a pair of nodes is not possible. Constraints (26) require that the total number of arcs is $n-1$, which is a known fact for a tree (e.g., Ahuja et al., 1993). Constraints (27) give the zero/one restrictions on the variables $w_{i c}$ and $w_{i l}$.

We remark that constraints (15)-(26) address topological requirements other than the hop requirement. Accordingly, constraints (15)-(26) provide a valid topology-enforcing formulation only when combined with a set of constraints that imposes the hop requirement, as noted in (14). When MTZ constraints are used as sub-tour elimination constraints, any of the constraints (6) and (9)-(11) can be used for this purpose. We note that any other set of hop-enforcing constraints, determined on the basis of the type of sub-tour elimination constraints used (such as flow-based constraints), can also be used with modifications as necessary. In this regard, constraints (15)-(26) are not particular to a formulation in which only MTZ constraints are used.
The distinction between central nodes and leaf nodes is used earlier in Akgun and Tansel (2010) in the context of the Minimum-Degree Constrained MST Problem (MDCMST) where the purpose is to find a minimum spanning tree such that each non-leaf node has at least $d$ nodes incident to it and in Akgun and Tansel (2009) in the context of the Degree-Constrained MST Problem (DCMST) where the degree constraint is on the upper bound rather than on the lower bound. The need to make that distinction is explicit in MDCMST from the problem itself while it is not in DCMST and in HMST. In this regard, using the distinction in DCMST and HMST is new, which is motivated by the successful results obtained for MDCMST. As stated in this paper and in the other two studies, some constraints based on the aforementioned distinction are valid for all spanning tree problems with restrictions and hence some constraints are common in all three studies. However, the underlying discussions for the three problems are different and hence there occurs a need to adapt some of the constraints to the problem structure. For example, constraints (17)-(21), a part of the topology-enforcing constraints, are different or expressed differently in all three studies to better utilize the problem structure. The same applies to the MTZ-based constraints (28)-(31) (Section 3.2) that also depend on this distinction. The computational results show that the proposed models for all problems take advantage of the constraints based on this distinction and produce improved LP bounds and solution times. Specifically, the models developed for MDCMST and DCMST, for which MTZ-based constraints are used the first time, are better than the flow-based models with respect to solution times while they are not better than but competitive with the flow-based models. The models developed for HMST in this paper are better than previous MTZ-based models in the literature with respect to both LP bounds and solution times.

\subsection{Improved MTZ constraints (IMTZ)}

We now give an improved set of MTZ constraints that take advantage of the distinction between central and leaf nodes as well as the greater degrees of freedom in choosing node labels in spanning trees than in TSP tours.

In a feasible solution of the HMST problem, each node is either a central node or a leaf node. Because a non-root leaf node has one incoming arc whose origin is necessarily a central node, then the monotonicity of node labels can be maintained by requiring that the labels of all non-root leaf nodes be greater than the highest possible label that can be assigned to central nodes. This condition is easily fulfilled if we assign the label value $p$ to each non-root leaf node while permitting central nodes to take label values of at most $p-1$. If all nodes other than the root node are leaf nodes, then the root node receives the node label of 0 and all other nodes receive node labels of $p$. If there is a non-root central node, then its label will be between 1 and $p-1$. Thus, in finding feasible solutions for the HMST problem, looking only for solutions in which the label values of non-root leaf nodes are restricted to $p$ and the label values of central nodes are restricted to values less than or equal to $p-1$ is sufficient. This can be achieved by adding the following constraints:

$u_{i} \geqslant p w_{i l} \quad i \in(V-r)$,

$u_{i} \leqslant p-w_{i c} \quad i \in(V-r)$.

Constraints (28) together with an appropriate hop constraint require that the labels of all non-root leaf nodes equal $p$. Constraints (29) restrict the labels of central nodes to be at most $p-1$. We take constraints (2)-(5) together with constraints (28) and (29) as the improved set of MTZ constraints and abbreviate it as IMTZ. 
Notice that because constraints (10) and (11) require $u_{i}$ and $u_{j}$ to differ exactly by one if edge $\{i, j\}$ is in the solution, using constraints (28) with constraints (10) and (11) may cause infeasibility. Hence, constraints (28) can only be used in conjunction with constraints (6) and (9).

Consider a solution in which the root $r$ is a central node and a leaf node $i$ is directly connected to the root. Constraints (12) require node $i$ to take on a label value of 1 while constraints (28) require node $i$ to take on a label value of $p$. To correct this inconsistency and be consistent with constraints (28), we modify constraints (12) and (13) as follows:

$u_{j} \leqslant p-(p-1) x_{i j}+(p-1) w_{j l} \quad i=r, j \in(V-r)$,

$u_{j} \geqslant 2-x_{i j}+(p-2) w_{j l} \quad i=r, j \in(V-r)$.

Constraints (30) require that the value of $u_{j}$ be equal to one if node $j$ is a central node and $x_{r j}=1$ and that the value of $u_{j}$ be less than or equal to $p$ if node $j$ is a leaf node and $x_{r j}=1$. Constraints (31) ensure that the value of $u_{j}$ is greater than or equal to 2 if node $j$ is a central node and $x_{r j}=0$ and that the value of $u_{j}$ is greater than or equal to $p$ if node $j$ is a leaf node and $x_{r j}=0$. In all other cases, constraints (30) and (31) become redundant.

\subsection{New formulations for the HMST problem}

We give now the new formulations for the HMST problem obtained by combining the constraint sets BMTZ, IMTZ, and ITEF with liftings offered by Gouveia (1995). Recall that constraints (28) can only be coupled with constraints (6) and (9).

BMTZ/ITEF: Model with Basic MTZ Constraints/Improved Topology Enforcing Constraints

(1)-(8) and (15)-(27)

IMTZ/ITEF: Model with Improved MTZ Constraints/Improved Topology Enforcing Constraints

(1)-(5), (9), (7), (8) and (15)-(29).

IMTZ/E1ITEF: Extension 1 of IMTZ/ITEF

(1), (2), (4), (5), (7), (8), (9), and (1)-(8), (8), (10)-(29).

IMTZ/E2ITEF: Extension 2 of IMTZ/ITEF

(1), (2), (4), (5), (7), (8), (10), (15)-(27), and (29).

IMTZ/E3ITEF: Extension 3 of IMTZ/ITEF

(1), (2), (4), (5), (7), (8), (11), (15)-(27), and (29).

IMTZ/E4ITEF: Extension 4 of IMTZ/ITEF

(1), (2), (4), (5), (7), (8), (10), (11), (15)-(27), and (29).

IMTZ/E5ITEF: Extension 5 of IMTZ/ITEF

(1), (2), (4), (5), (7), (8), (9), and (15)-(31).

IMTZ/E6ITEF: Extension 6 of IMTZ/ITEF

(1), (2), (4), (5), (7), (8), (10), (15)-(27), and (29)-(31).

IMTZ/E7ITEF: Extension 7 of IMTZ/ITEF

(1), (2), (4), (5), (7), (8), (11), (15)-(27), and (29)-(31).

IMTZ/E8ITEF: Extension 8 of IMTZ/ITEF

(1), (2), (4), (5), (7), (8), (10), (11), (15)-(27), and (29)-(31).

BMTZ/ITEF is defined to see the effects of the new MTZ constraints (28) and (29) that are included in IMTZ/ITEF but not in BMTZ/ITEF. The new model IMTZ/ITEF corresponds to the basic model BMTZ/BTEF of Gouveia (1995) while the new model IMTZ/ EiITEF corresponds to the model BMTZ/EiBTEF of Gouveia (1995) for $\boldsymbol{i}=\mathbf{1}, \ldots, \mathbf{8}$.

We also propose the following relaxed model, Rel-M, which gives the best performance of all existing and new models in terms of solution times. Regarding the LP bounds, Rel-M generally gives better than or as good LP bounds as the existing models BMTZ/ BTEF, and BMTZ/EiBTEF, $\boldsymbol{i}=\mathbf{1}, \ldots, \mathbf{8}$, while it generally gives worse LP bounds than the proposed models IMTZ/ITEF, and IMTZ/EiITEF, $\boldsymbol{i}=\mathbf{1}, \ldots, \mathbf{8}$. The primary reason for considering $\mathbf{R e l - M}$ is its significant success in computation times.
Rel-M is obtained from the model IMTZ/ITEF by omitting constraints (19) and (21) from it while adding constraints (31) and (32) to it.

Rel-M: Relaxed Model for the HMST problem

In addition to (1)-(8), (15)-(18), (20), (22)-(29), and (31)

$\sum_{j \neq r} x_{i j} \geqslant w_{i l}-1 \quad i \in(V-r)$.

The exclusion of constraints (19) and (21) from Rel-M permits the variables $w_{i c}$ and $w_{i l}$ to take on values of 1 and 0 , respectively, when a non-root node $i$ is in fact a leaf node. Even though this does not agree with the intended meaning attached to these variables, such solutions nevertheless define feasible trees. We take a more liberal interpretation of the auxiliary variables $w_{i c}$ and $w_{i l}$ in Rel$\mathbf{M}$ and interpret them as node labels that generally distinguish central nodes from leaf nodes but sometimes with incorrect values.

Note that the left sides of constraints (21) and (32) are the same and specify the total number of outgoing arcs at a non-root node while the right sides of (21) and (32) are the negatives of each other $\left(1-w_{i l}\right.$ and $\left.w_{i l}-1\right)$. Accordingly, we may think of (32) as a replacement for (21). We do not, however, recommend the use of (32) in place of (21) in the proposed models other than Rel-M as its use in these models generally worsens the solution times while its use in Rel-M improves the solution times.

\section{Test problems}

Computational tests are performed using specially-structured test problems from the literature (e.g., Dahl et al., 2006). Test problems consist of three 20 -node, three 40 -node, and three 60 -node, complete networks with 210, 820, and 1830 edges, respectively. For each network size, two Euclidean instances, TC and TE, and one random instance, TR, are considered. Euclidean instances differ from each other based on the location of the root node. In TC instances, the root node is located in the center of the grid while in TE instances the root node is located at a corner of the grid. In the following, a test instance(s) is referred to with the type of the instance and the number of the nodes in the network, e.g., TE $\mathbf{2 0}$ refers to TE instance with $\mathbf{2 0}$ nodes. For each instance, the hop parameter $p$ is set to 3,4 , and 5 .

The size of each instance is reduced by applying an arcelimination test used in the literature (e.g., Gouveia, 1996; Dahl et al., 2006). The test is based on the fact that if $c_{i j}>c_{r j}$, then no optimal solution uses arc $(i, j)$ and if $c_{i j}=c_{r j}(i \neq r)$, then there is an optimal solution that does not include arc $(i, j)$. Thus, arc $(i, j)$ can be eliminated whenever $c_{i j} \geqslant c_{r j}$. The number of arcs remaining after the elimination test in $\mathbf{2 0}$-node, $\mathbf{4 0}$-node, and $\mathbf{6 0}$-node networks for TC, TE, and TR instances are $38 \%, 82 \%$, and $52 \%, 33 \%$, $75 \%$, and $51 \%$, and $31 \%, 74 \%$, and $58 \%$ of the original numbers, respectively. The results show that the best (worst) results are obtained with TC (TE) instances implying that TE instances will be much more difficult to solve than the other two types of instances.

Computational tests are performed on a PC with a $3.0 \mathrm{GHz}$ Intel Core 2 Duo processor and 3 GB of RAM by using ILOG CPLEX 9.0. The models are run until optimality is attained or for 10 hours (36,000 CPU seconds) at maximum and by using default settings of CPLEX (e.g., moving the best bound strategy for branching is used, cuts are allowed) except that file storage is set to 3 , which allows tree file to be stored on the hard disk when it reaches the default limit in order not to run out of memory (ILOG CPLEX, 2003).

In the tables presenting computational studies, LP relaxation bounds, run times, optimal objective function values (when available), and best feasible objective function values together with relative optimality gaps are given. The relative optimality gap is defined as $|B P-B F| /\left(10^{-10}+|B P|\right)$ where $B P$ is the objective 
function value of the best integer solution and $B F$ is the best remaining objective function value of any unexplored node (ILOG CPLEX, 2003).

The bold and underlined values in each row of the tables indicate the best and worst results (with respect to solution time if optimality is attained and optimality gap otherwise), respectively, for the corresponding problem. All non-bracketed values in the [BP-Gap\%] columns are the optimal objective function values. A "[ ]" in a [BP-Gap\%] column indicates that the model could not be solved optimally within 36,000 seconds. In this case, the first and second figures separated by a dash in a bracket are the values of BP and the relative optimality gap, respectively.

\section{LP bounds}

We take up the discussion regarding the LP relaxation bounds in three parts. In the first and second parts, we discuss the relative standing of the existing models and proposed models among themselves, respectively. In the third part, we discuss the relative standing of the existing and new models with respect to each other. In this discussion, $P_{L}$ is used to represent the optimal objective function value of the LP relaxation of a linear integer programming problem $P$.

Table 1 gives the LP bounds for the existing models studied in Gouveia (1995). The results show that $\mathbf{B M T Z} / \mathbf{B T E F}_{\mathbf{L}}=\mathbf{B M T Z}$ E1BTEF $_{\mathbf{L}}$ for all instances, i.e., replacing constraints (3) and (6) with constraints (9) does not improve the LP bounds. This is partially in compliance with the results in Gouveia (1995) where there are in-

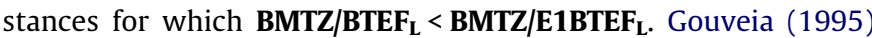

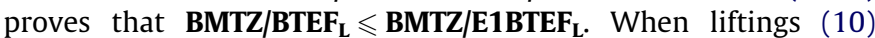
and (11) are used instead of constraints (9) separately, they provide relatively good improvements in the LP bounds of BMTZ/BTEF (BMTZ/E1BTEF). The improvements change from 3.3\% to $15.45 \%$ with an average of $8.3 \%$ and from $3.85 \%$ to $15.45 \%$ with an average of $8.4 \%$ for constraints (10) and (11), respectively. So, BMTZ/ E1BTEF $_{\mathbf{L}}<$ BMTZ/E2BTEF $_{\mathbf{L}}$ and BMTZ/E1BTEF $_{\mathbf{L}}<$ BMTZ/E3BTEF $_{\mathbf{L}}$ for all instances. However, Gouveia (1995) reports instances where the strict inequality holds at equality. Liftings (10) and (11) do not dominate each other because there are instances for which BMTZ/ E2BTEF $_{\mathbf{L}}<\mathbf{B M T Z}_{\mathbf{E}} \mathbf{E B B T E F}_{\mathbf{L}}$ (e.g., Problems 1-3) and BMTZ/ E3BTEF $_{\mathbf{L}}<$ BMTZ/E2BTEF $_{\mathbf{L}}$ (e.g., Problems 9-12). When constraints (10) and (11) are used together, a slight improvement over both BMTZ/E2BTEF $_{\mathbf{L}}$ and $\mathbf{B M T Z / E 3 B T E F}_{\mathbf{L}}$ is attained for Problems 7, 8, 10, and 16-18. For other instances, BMTZ/E4BTEF $=$ Max $\{$ BMTZ E2BTEF $_{\mathbf{L}}$, BMTZ/E3BTEF $\left._{\mathbf{L}}\right\}$. Thus, BMTZ/E2BTEF $\mathbf{L} \leqslant$ BMTZ/E4BTEF $_{\mathbf{L}}$

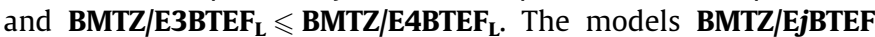
for $\boldsymbol{j}=\mathbf{5 , 6 , 7 , 8}$ obtained by adding liftings (12) and (13) toBMTZ/ EiBTEF for $\boldsymbol{i}=\boldsymbol{j}-\mathbf{4}$, respectively, give the same or slightly better LP bounds than their corresponding ones. Specifically, BMTZ/ E1BTEF $_{\mathbf{L}} \leqslant$ BMTZ/E5BTEF $_{\mathrm{L}} \quad$ BMTZ/E2BTEF $_{\mathrm{L}} \leqslant$ BMTZ/E6BTEF $_{\mathrm{L}}$, BMTZ/E3BTEF $_{\mathbf{L}} \leqslant$ BMTZ/E7BTEF $_{\mathbf{L}}$, and BMTZ/E4BTEF $_{\mathbf{L}} \leqslant \mathbf{B M T Z}^{-}$ E8BTEF $_{\mathbf{L}}$. The foregoing comments regarding the relative standing of the models BMTZ/EiBTEF for $\boldsymbol{i}=\mathbf{1 , 2 , 3 , 4}$ remain valid for the corresponding models BMTZ/EjBTEF for $\boldsymbol{j}=\mathbf{5 , 6 , 7 , 8}$. Of all the models, $\quad$ BMTZ/E8BTEF $_{\mathrm{L}} \geqslant$ BMTZ/EiBTEF $_{\mathrm{L}}, \boldsymbol{i}=\mathbf{1}, \ldots, \mathbf{7}$, andBMTZ/ $\mathbf{E B B T E F}_{\mathbf{L}} \geqslant \mathbf{B M T Z} / \mathbf{B T E F}_{\mathbf{L}}$. However, the differences between the LP bounds of BMTZ/E8BTEF and those of the other existing models are marginal except for BMTZ/BTEF, BMTZ/E1BTEF and BMTZ/ E5BTEF. Fig. 1 shows the relative standing of the models graphically.

Table 2 gives LP bounds for the proposed models. The results show that, even though marginal, the improved version of MTZ constraints is marginally stronger than the basic version of MTZ

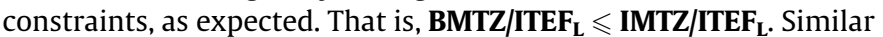
to the case of BMTZ/BTEF and BMTZ/E1BTEF, IMTZ/ITEF $_{\mathbf{L}}=\mathbf{I M T Z} /$
E1ITEF $_{\mathbf{L}}$ for all cases. That is, using constraints (9) in place of constraints (3) and (6) in the basic model IMTZ/ITEF does not improve the LP bounds for the given instances. When liftings (10) are used instead of constraints (3) and (6), there are cases for which IMTZ/ E1ITEF $_{\mathbf{L}}=$ IMTZ $_{\text {E2ITEF }}$ (Problems 6, 9, 10-12, and 18), cases for which IMTZ/E1ITEF $_{\mathbf{L}}<$ IMTZ/E2ITEF $_{\mathbf{L}}$ (Problems 1-2, 4-5, and 1317), and cases for which IMTZ/E1ITEF $_{\mathbf{L}}>$ IMTZ/E2ITEF $_{\mathbf{L}}$ (Problems 3,7 , and 8 ). This is contrary to the expectation that using constraints (10) will improve the LP bounds. IMTZ/E1ITEF $_{\mathbf{L}}>$ IMTZ/ E2ITEF $_{\mathbf{L}}$ results from the fact that constraints (10) in IMTZ/E2ITEF cannot compensate for the decrease in the LP bounds caused by not including constraints (28) in IMTZ/E2ITEF. (Remember that constraints (10) and (11) cannot be used with constraints (28) and hence constraints (28) are included in IMTZ/E1ITEF while they are not included in IMTZ/E2ITEF). The results show that IMTZ/ E1ITEF $_{\mathbf{L}} \leqslant \mathbf{I M T Z}_{\mathbf{E}} \mathbf{E}_{\mathbf{I T E F}}$ indicating that using constraints (11) improves the LP bounds and that constraints (11) can compensate for the lack of constraints (28). In compliance with the ongoing discussion, IMTZ/E2ITEF $_{\mathbf{L}} \leqslant$ IMTZ/E3ITEF $_{\mathbf{L}}=$ IMTZ/E4ITEF $_{\mathbf{L}}$. Thus, constraints (11) dominate constraints (10) for the given instances and using constraints (10) and (11) simultaneously in IMTZ/E4ITEF does not improve upon the LP bounds of IMTZ/E3ITEF with constraints (11). Note that these two results are not observed for the models of Gouveia (1995). Liftings (30) and (31), the new versions of constraints (12) and (13), are added to IMTZ/EiITEF for $\boldsymbol{i}=\mathbf{1 , 2 , 3 , 4}$, to obtain IMTZ/EjITEF for $\boldsymbol{j}=\boldsymbol{i}+\mathbf{4}$, respectively. Their effect on the LP bounds is similar to the one that constraints (12) and (13) have on the LP bounds of the models of Gouveia (1995). Specifically, $\quad$ IMTZ/E1ITEF $_{\mathbf{L}} \leqslant$ IMTZ/E5ITEF $_{\mathbf{L}}, \quad$ IMTZ/E2ITEF $_{\mathbf{L}} \leqslant$ IMTZ/ E6ITEF $_{L}, \quad$ IMTZ/E3ITEF $_{L} \leqslant$ IMTZ/E7ITEF $_{L}$, and IMTZ/E4ITEF IMTZ/E8ITEF L $_{\mathbf{L}}$. However, the improvements in the LP bounds are marginal. The relative standing of the models IMTZ/EjITEF for $\boldsymbol{j = 5 , 6 , 7 , 8}$ is IMTZ/E5ITEF $_{\mathbf{L}} \leqslant$ IMTZ/E6ITEF $_{\mathbf{L}} \leqslant$ IMTZ/E7ITEF $_{\mathbf{L}}=$ IMTZ/E8ITEF $_{\mathbf{L}}$. Note that constraints (10) together with constraints (30) and (31) can now compensate for the decrease in the LP bounds resulting from the lack of constraints (28). Of all the models, IMTZ/E7ITEF and IMTZ/E8ITEF give the best (and the same) LP bounds. Another important observation in Table 2 is that adding the liftings offered by Gouveia (1995) only slightly increases the LP bounds of IMTZ/ITEF. The differences between the best LP bounds (of IMTZ/E8ITEF) and those of IMTZ/ITEF change from 0\% to $2.47 \%$ with an average of $0.54 \%$. Finally, even though marginal, Rel-M $\mathbf{M}_{\mathbf{L}} \leq \mathrm{IMTZ}_{\mathbf{E}}$ EiITEF $_{\mathrm{L}}$ for $\boldsymbol{i}=\mathbf{3}, \ldots, \boldsymbol{8}$ for all instances. Rel-M is not dominated by BMTZ/ITEF, IMTZ/ITEF, IMTZ/E1ITEF, and IMTZ/ E2ITEF. There are instances for which the LP bounds of Rel-M are the same as, better than, and worse than those of BMTZ/ITEF, IMTZ/ITEF, IMTZ/E1ITEF, and IMTZ/E2ITEF. Fig. 2 shows the relative standing of the new models graphically.

We now compare the relative standing of the proposed models and the existing models. The comparison is made between the existing model BMTZ/BTEF (BMTZ/EiBTEF for $\boldsymbol{i}=\mathbf{1}, \ldots, \boldsymbol{8}$ ) and the corresponding, proposed model IMTZ/ITEF (IMTZ/EiITEF for $\boldsymbol{i}=\mathbf{1}, \ldots, \boldsymbol{8})$. The corresponding columns in Tables 1 and 2 show that

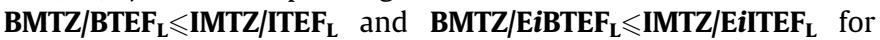
$\boldsymbol{i}=\mathbf{1}, \ldots, \boldsymbol{8}$ for all cases. The strict inequality holds for the basic models IMTZ/ITEF and BMTZ/BTEF as well as for the extended models IMTZ/EiITEF and BMTZ/EiBTEF with $\boldsymbol{i}=\mathbf{1 , 5}$. The differences between the LP bounds of these pairs of new and existing models change from 3.64\% (Problem 1 for IMTZ/ITEF and BMTZ/ BTEF pair) to 22\% (Problem 6 for all three pairs). For other models in which constraints (10) and (11) are used, the differences between the LP bounds are smaller. An increase of at most 7\% is attained for Problem 4 between all pairs of new and existing models. The highest increases are attained for TE 20 instances (from 6\% to 7\%). Clearly, constraints (10) and (11) contribute significantly to the improvement of LP bounds of the models in Gouveia 
Table 1

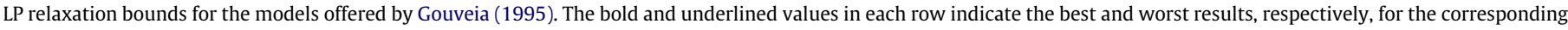
problem.

\begin{tabular}{|c|c|c|c|c|c|c|c|c|c|c|c|}
\hline Pr. id. & Pr. type & $p$ & $\begin{array}{l}\text { BMTZ/ } \\
\text { BTEF }\end{array}$ & $\begin{array}{l}\text { BMTZ/ } \\
\text { E1BTEF }\end{array}$ & $\begin{array}{l}\text { BMTZ/ } \\
\text { E2BTEF }\end{array}$ & $\begin{array}{l}\text { BMTZ/ } \\
\text { E3BTEF }\end{array}$ & $\begin{array}{l}\text { BMTZ/ } \\
\text { E4BTEF }\end{array}$ & $\begin{array}{l}\text { BMTZ/ } \\
\text { E5BTEF }\end{array}$ & $\begin{array}{l}\text { BMTZ/ } \\
\text { E6BTEF }\end{array}$ & $\begin{array}{l}\text { BMTZ/ } \\
\text { E7BTEF }\end{array}$ & $\begin{array}{l}\text { BMTZ/ } \\
\text { E8BTEF }\end{array}$ \\
\hline 1 & TC 20 & 3 & 294.67 & 294.67 & 304.38 & 306.00 & 306.00 & 294.67 & 305.56 & 306.00 & 306.00 \\
\hline 2 & & 4 & 290.00 & 290.00 & 302.75 & 303.71 & 303.71 & 290.00 & 303.50 & 303.71 & 303.71 \\
\hline 3 & & 5 & $\underline{289.20}$ & $\underline{289.20}$ & 302.00 & 302.75 & 302.75 & $\underline{289.20}$ & 302.67 & 302.75 & 302.75 \\
\hline 4 & TE 20 & 3 & 258.67 & 258.67 & 284.00 & 284.00 & 284.00 & 261.33 & 284.00 & 284.00 & 284.00 \\
\hline 5 & & 4 & $\underline{248.00}$ & $\underline{248.00}$ & 284.00 & 284.00 & 284.00 & $\underline{248.00}$ & 284.00 & 284.00 & 284.00 \\
\hline 6 & & 5 & $\underline{246.00}$ & $\underline{246.00}$ & 284.00 & 284.00 & 284.00 & 246.00 & 284.00 & 284.00 & 284.00 \\
\hline 7 & TR 20 & 3 & $\underline{128.67}$ & $\underline{128.67}$ & 138.14 & 139.50 & 140.17 & 128.67 & 139.62 & 139.50 & 140.17 \\
\hline 8 & & 4 & 124.50 & 124.50 & 137.00 & 137.06 & 138.14 & 124.50 & 138.00 & 137.06 & 138.14 \\
\hline 9 & & 5 & $\underline{122.40}$ & $\underline{122.40}$ & 137.00 & 136.23 & 137.00 & $\underline{122.40}$ & 137.00 & 136.23 & 137.00 \\
\hline 10 & TC 40 & 3 & $\underline{446.00}$ & $\underline{446.00}$ & 472.00 & 469.17 & 472.50 & $\underline{446.00}$ & 472.50 & 469.17 & 472.50 \\
\hline 11 & & 4 & $\underline{437.00}$ & $\underline{437.00}$ & 472.00 & 470.00 & 472.00 & 437.00 & 472.00 & 470.00 & 472.00 \\
\hline 12 & & 5 & $\underline{435.20}$ & $\underline{435.20}$ & 472.00 & 470.57 & 472.00 & $\underline{435.20}$ & 472.00 & 470.57 & 472.00 \\
\hline 13 & TE 40 & 3 & $\underline{458.67}$ & $\underline{458.67}$ & 481.78 & 485.06 & 485.06 & 461.00 & 484.58 & 485.06 & 485.54 \\
\hline 14 & & 4 & $\underline{456.00}$ & $\underline{456.00}$ & 481.00 & 481.78 & 481.78 & $\underline{456.00}$ & 481.65 & 481.78 & 481.78 \\
\hline 15 & & 5 & $\underline{455.60}$ & $\underline{455.60}$ & 480.36 & 481.00 & 481.00 & $\underline{455.60}$ & 480.91 & 481.00 & 481.00 \\
\hline 16 & TR 40 & 3 & $\underline{117.33}$ & $\underline{117.33}$ & 127.07 & 127.75 & 129.17 & 118.11 & 128.62 & 127.75 & 129.17 \\
\hline 17 & & 4 & $\underline{114.50}$ & $\underline{114.50}$ & 126.50 & 126.03 & 127.07 & $\underline{114.50}$ & 127.06 & 126.03 & 127.07 \\
\hline 18 & & 5 & $\underline{113.40}$ & $\underline{113.40}$ & 126.00 & 125.73 & 126.50 & $\underline{113.40}$ & 126.50 & 125.73 & 126.50 \\
\hline
\end{tabular}

$\square \mathrm{B} / \mathrm{B} \square \mathrm{B} / \mathrm{E} 1 \mathrm{~B} \square \mathrm{B} / \mathrm{E} 2 \mathrm{~B} \square \mathrm{B} / \mathrm{E} 3 \mathrm{~B} \square \mathrm{B} / \mathrm{E} 4 \mathrm{~B} \square \mathrm{B} / \mathrm{E} 5 \mathrm{~B} \square \mathrm{B} / \mathrm{E} 6 \mathrm{~B} \square \mathrm{B} / \mathrm{E} 7 \mathrm{~B} \square \mathrm{B} / \mathrm{E} 8 \mathrm{~B}$

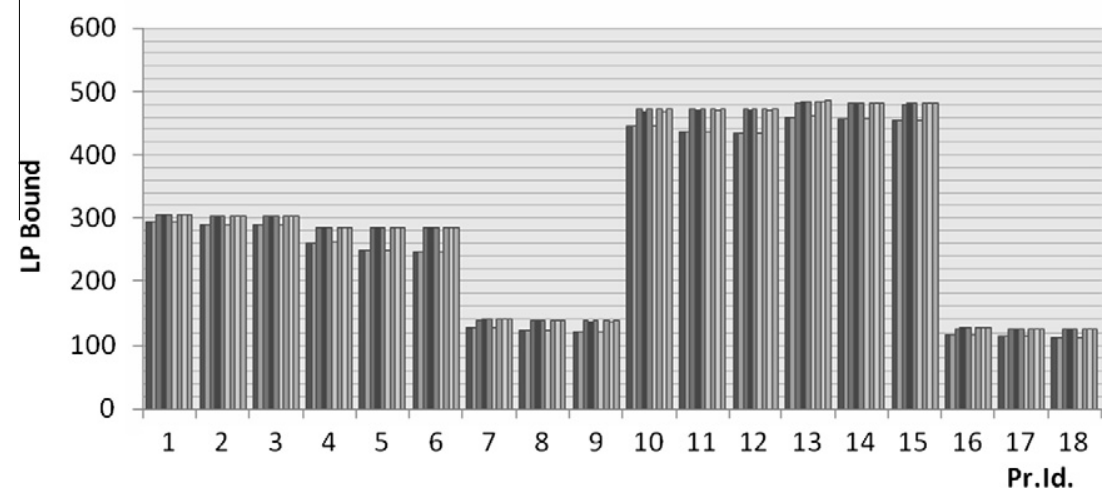

Fig. 1. The relative standing of the models studied in Gouveia (1995) with respect to LP bounds.

(1995). The contribution of liftings (30) and (31) to LP bounds is miniscule when used with the proposed models.

To better assess the contribution of the new models, we now compare BMTZ/E8BTEF (the existing model with the best LP bounds) and IMTZ/ITEF (the new model with the worst LP bounds).

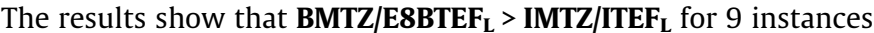
(Problems 1-3, 7-8, 10, and 16-18), $\mathbf{B M T Z / E 8 B T E F}_{\mathbf{L}}<\mathbf{I M T Z}_{\mathbf{I T E F}}$ for 6 instances (Problems $4-6$ and 13-15), and $\mathbf{B M T Z} / \mathbf{E B B T E F}_{\mathbf{L}}=$ IMTZ/ITEF $_{\mathbf{L}}$ for 3 instances (Problems 9 and 11-12). The increases in the LP bounds change from 0.3\% (0.5 units for Problem 18) to $2 \%$ (2.49 units for Problem 16) for the cases with BMTZ/E8BTE$\mathbf{F}_{\mathbf{L}}>$ IMTZ/ITEF $_{\mathbf{L}}$ while the increases in the LP bounds change from $0.38 \%$ (1.86 units for Problem 13) to 6.4\% (18.36 units for Problem

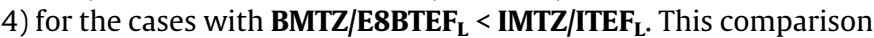
reveals an important finding: The proposed basic model IMTZ/ITEF provides as good or better LP bounds in most cases as the strongest existing model BMTZ/E8BTEF while it provides marginally worse bounds in some cases. That is, our computational tests provide good empirical evidence that the model IMTZ/ITEF is at least as competitive as the strongest existing model BMTZ/E8BTEF. Note that IMTZ/ITEF does not include any of the liftings considered in Gouveia (1995) while BMTZ/E8BTEF includes all of the liftings (10)-(13).

We finally note that the relaxed model Rel-M, which is the best one in terms of solution times, is also in competition in LP bounds with the strongest existing model BMTZ/E8BTEF. Rel-M produces the same or better LP bounds than BMTZ/E8BTEF for 8 instances with the highest difference being 6\% (17 units) for TE 20 instances. Rel-M supplies marginally worse bounds than BMTZ/E8BTEF for 10 instances with the highest difference being $0.89 \%$ (1.15 units) for Problem 16.

\section{Solution times and optimality gaps}

Tables 3 and 4 give solution times, optimal objective function values (if optimal solution is attained), and the best feasible objective function values together with relative optimality gaps (if optimal solution is not available) for the models offered by Gouveia (1995) and the proposed models in this paper, respectively. 
Table 2

LP relaxation bounds for the new models. The bold and underlined values in each row indicate the best and worst results, respectively, for the corresponding problem.

\begin{tabular}{|c|c|c|c|c|c|c|c|c|c|c|c|c|c|}
\hline $\begin{array}{l}\text { Pr. } \\
\text { id. }\end{array}$ & $\begin{array}{l}\text { Pr. } \\
\text { type }\end{array}$ & $p$ & $\begin{array}{l}\text { BMTZ/ } \\
\text { ITEF }\end{array}$ & $\begin{array}{l}\text { IMTZ/ } \\
\text { ITEF }\end{array}$ & $\begin{array}{l}\text { IMTZ/ } \\
\text { E1ITEF }\end{array}$ & $\begin{array}{l}\text { IMTZ/ } \\
\text { E2ITEF }\end{array}$ & $\begin{array}{l}\text { IMTZ/ } \\
\text { E3ITEF }\end{array}$ & $\begin{array}{l}\text { IMTZ/ } \\
\text { E4ITEF }\end{array}$ & $\begin{array}{l}\text { IMTZ/ } \\
\text { E5ITEF }\end{array}$ & $\begin{array}{l}\text { IMTZ/ } \\
\text { E6ITEF }\end{array}$ & $\begin{array}{l}\text { IMTZ/ } \\
\text { E7ITEF }\end{array}$ & $\begin{array}{l}\text { IMTZ/ } \\
\text { E8ITEF }\end{array}$ & Rel-M \\
\hline 1 & TC 20 & 3 & 304.06 & 305.39 & 305.39 & 305.71 & 307.00 & 307.00 & 306.00 & 307.00 & 307.00 & 307.00 & 306.00 \\
\hline 2 & & 4 & 302.57 & 302.57 & 302.57 & 302.75 & 303.71 & 303.71 & 303.00 & 303.50 & 303.71 & 303.71 & 303.00 \\
\hline 3 & & 5 & $\overline{302.07}$ & $\overline{302.07}$ & $\overline{302.07}$ & $\underline{302.00}$ & 302.75 & 302.75 & 302.40 & 302.67 & 302.75 & 302.75 & 302.40 \\
\hline 4 & TE 20 & 3 & 302.33 & 302.36 & 302.36 & 302.60 & 303.29 & 303.29 & 302.67 & 303.11 & 303.67 & 303.67 & $\underline{301.00}$ \\
\hline 5 & & 4 & 301.50 & 301.50 & 301.50 & 301.67 & 301.80 & 301.80 & 301.50 & 301.67 & 301.80 & 301.80 & 301.00 \\
\hline 6 & & 5 & 301.00 & 301.00 & 301.00 & 301.00 & 301.00 & 301.00 & 301.00 & 301.00 & 301.00 & 301.00 & $\overline{301.00}$ \\
\hline 7 & TR 20 & 3 & $\underline{137.89}$ & 138.52 & 138.52 & 138.33 & 140.70 & 140.70 & 139.62 & 140.00 & 140.70 & 140.70 & 139.62 \\
\hline 8 & & 4 & $\overline{137.00}$ & 137.27 & 137.27 & 137.00 & 138.14 & 138.14 & 137.89 & 138.14 & 138.33 & 138.33 & 137.89 \\
\hline 9 & & 5 & $\overline{137.00}$ & 137.00 & 137.00 & $\overline{137.00}$ & 137.00 & 137.00 & 137.00 & 137.00 & 137.00 & 137.00 & 137.00 \\
\hline 10 & TC 40 & 3 & $\underline{472.00}$ & $\underline{472.00}$ & $\underline{472.00}$ & 472.00 & 472.50 & 472.50 & 472.50 & 472.67 & 472.67 & 472.67 & 472.50 \\
\hline 11 & & 4 & $\overline{472.00}$ & $\overline{472.00}$ & $\overline{472.00}$ & $\overline{472.00}$ & 472.00 & 472.00 & 472.00 & 472.00 & 472.00 & 472.00 & 472.00 \\
\hline 12 & & 5 & 472.00 & 472.00 & 472.00 & 472.00 & 472.00 & 472.00 & 472.00 & 472.00 & 472.00 & 472.00 & 472.00 \\
\hline 13 & TE 40 & 3 & 487.33 & 487.40 & 487.40 & 487.83 & 488.75 & 488.75 & 488.67 & 489.42 & 489.86 & 489.86 & $\underline{484.10}$ \\
\hline 14 & & 4 & 486.63 & 486.65 & 486.65 & 487.02 & 487.78 & 487.78 & 487.06 & 487.69 & 487.83 & 487.83 & $\overline{481.06}$ \\
\hline 15 & & 5 & 486.20 & 486.20 & 486.20 & 486.35 & 487.00 & 487.00 & 486.53 & 486.93 & 487.02 & 487.02 & $\overline{480.53}$ \\
\hline 16 & TR 40 & 3 & 126.67 & 126.68 & 126.68 & 127.16 & 129.33 & 129.33 & 128.02 & 129.24 & 129.80 & 129.80 & 128.02 \\
\hline 17 & & 4 & 126.25 & 126.25 & 126.25 & 126.50 & 127.07 & 127.07 & 126.50 & 127.15 & 127.16 & 127.16 & 126.50 \\
\hline 18 & & 5 & $\underline{126.00}$ & $\underline{126.00}$ & 126.00 & $\underline{126.00}$ & 126.50 & 126.50 & 126.20 & 126.50 & 126.50 & 126.50 & 126.20 \\
\hline
\end{tabular}

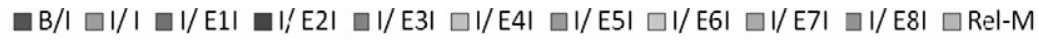

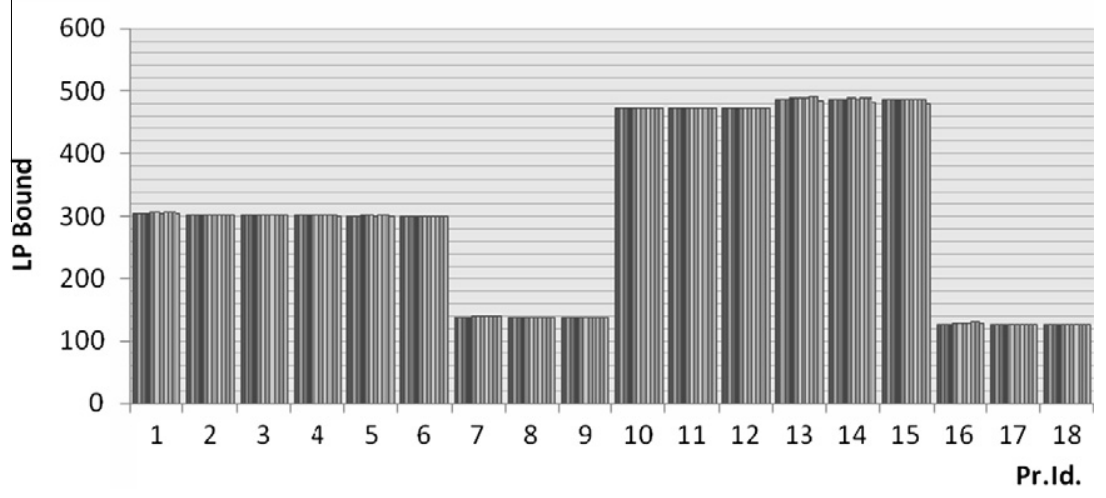

Fig. 2. The relative standing of the new models with respect to LP bounds.

The results show that all TC $\mathbf{2 0}$ and TR 20 instances are all solved in less than 1 second by all existing and new models. All TE 20 instances can be solved by the proposed models. The best solution time of 74.89 seconds is obtained by IMTZ/E7ITEF while the worst average solution time of 1106 seconds is obtained by IMTZ/E2ITEF (IMTZ/ITEF). Average solution times of 86.3 seconds and 116.87 seconds, which can be considered good, are obtained by IMTZ/E6ITEF and Rel-M, respectively. The average solution times of the other new models change from 127.57 seconds (IMTZ/E5ITEF) to 905.33 seconds (IMTZ/E3ITEF).

The existing models cannot solve all TE 20 instances (BMTZ/ E1BTEF cannot solve Problems 4 and 5 and BMTZ/E2BTEF cannot solve Problem 4). The average solution times of existing models other than BMTZ/E1BTEF and BMTZ/E2BTEF change from $90 \mathrm{sec}-$ onds (BMTZ/E7BTEF) to 7446 seconds (BMTZ/BTEF). With average solution times of 150 seconds and 253 seconds, BMTZ/E6BTEF and BMTZ/E8ITEF also produce good solution times.

TR 40 instances can be solved by all new models. The average solution times change from 6.5 seconds (Rel-M) to 5525 seconds IMTZ/ITEF (IMTZ/E1ITEF). The average solution times for Rel-M and IMTZ/EiITEF for $\boldsymbol{i}=\mathbf{5 , 6 , 7 , 8}$ are $6.5,16.38,32.13,33.58$, and
48.23 seconds, respectively. Note that these are the models with constraints (30) and (31), which are the adapted versions of constraints (12) and (13) in the existing models. Of the models without constraints (30) and (31), i.e., IMTZ/EiITEF for $\boldsymbol{i}=\mathbf{1 , 2 , 3 , 4}$ and IMTZ/ITEF, IMTZ/E4ITEF produces the best average solution time of 845 seconds (compared to the worst solution time of $48.23 \mathrm{sec}-$ onds). All TR 40 instances cannot be solved by the existing models (BMTZ/E1BTEF and BMTZ/E2ITEF cannot solve Problem 16). The average solution times of the remaining models change from 10.12 seconds (BMTZ/E7BTEF) to 1488 seconds (BMTZ/BTEF). As in the case of the new models with constraints (30) and (31), using constraints (12) and (13) significantly improves the solution times. The average solution times of BMTZ/EiBTEF for $\boldsymbol{i}=\mathbf{5 , 6 , 7 , 8}$ are under 22 seconds.

Regarding TC 40 instances, the new models with constraints (30) and (31), i.e., Rel-M and IMTZ/EiITEF for $\boldsymbol{i}=\mathbf{5 , 6 , 7 , 8}$ can solve all instances. Of these models, Rel-M and IMTZ/E8ITEF produce the best and worst average solution times of 803 seconds and 10,391 seconds, respectively. That the second best average solution time for these models is 3561 seconds (IMTZ/E7ITEF) indicates that Rel-M significantly dominates the other models. Of the mod- 
Table 3

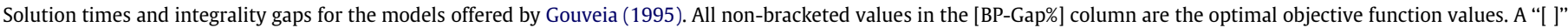

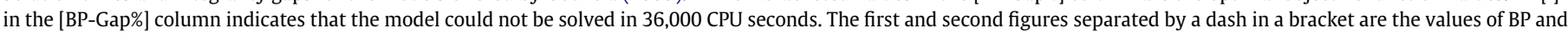

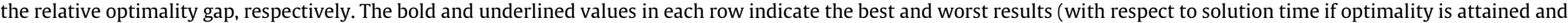
optimality gap otherwise), respectively, for the corresponding problem.

\begin{tabular}{|c|c|c|c|c|c|c|c|c|c|c|c|c|c|c|c|}
\hline \multirow[t]{2}{*}{ Pr. id. } & \multirow[t]{2}{*}{ Pr. type } & \multirow[t]{2}{*}{$p$} & \multicolumn{2}{|l|}{ BMTZ/BTEF } & \multicolumn{3}{|c|}{ BMTZ/E1BTEF } & \multicolumn{2}{|l|}{ BMTZ/E2BTEF } & \multicolumn{3}{|l|}{ BMTZ/E3BTEF } & \multicolumn{3}{|c|}{ BMTZ/E4BTEF } \\
\hline & & & [BP-Gap\%] & $\begin{array}{l}\text { Time } \\
\text { (seconds) }\end{array}$ & \multicolumn{2}{|c|}{ [BP-Gap\%] } & $\begin{array}{l}\text { Time } \\
\text { (seconds) }\end{array}$ & [BP-Gap\%] & $\begin{array}{l}\text { Time } \\
\text { (seconds) }\end{array}$ & [BP-Gap\%] & \multicolumn{2}{|c|}{$\begin{array}{l}\text { Time } \\
\text { (seconds) }\end{array}$} & \multicolumn{2}{|c|}{ [BP-Gap\%] } & $\begin{array}{l}\text { Time } \\
\text { (seconds) }\end{array}$ \\
\hline 1 & \multirow[t]{3}{*}{ TC 20} & 3 & 340 & 0.28 & \multicolumn{2}{|l|}{$\underline{340}$} & 4.27 & 340 & 3.01 & 340 & \multicolumn{2}{|c|}{0.45} & \multicolumn{2}{|l|}{340} & 0.45 \\
\hline 2 & & 4 & 318 & 0.05 & \multicolumn{2}{|l|}{$\overline{318}$} & $\underline{0.36}$ & 318 & 0.14 & 318 & \multicolumn{2}{|l|}{0.14} & 318 & & 0.16 \\
\hline 3 & & 5 & 312 & 0.02 & $\underline{312}$ & & $\underline{0.08}$ & 312 & 0.09 & 312 & 0.05 & & 312 & & 0.06 \\
\hline 4 & TE 20 & 3 & 449 & 11132 & [449- & $.73]$ & $\underline{36,000}$ & [458-11.35] & 36,000 & 449 & 194 & & 449 & & 1402 \\
\hline 5 & & 4 & 385 & 2741 & [385- & & $\overline{36,000}$ & 385 & 11697 & 385 & 931 & & 385 & & 2558 \\
\hline 6 & & 5 & 366 & 8466 & $\underline{366}$ & & $\underline{16401}$ & 366 & 2572 & 366 & 110 & & 366 & & 2454 \\
\hline 7 & TR 20 & 3 & 168 & 0.03 & 168 & & 0.25 & 168 & 0.25 & 168 & 0.06 & & 168 & & 0.09 \\
\hline 8 & & 4 & 146 & 0.02 & 146 & & 0.05 & 146 & 0.06 & 146 & 0.03 & & 146 & & 0.03 \\
\hline 9 & & 5 & 137 & 0.02 & 137 & & 0.00 & 137 & 0.01 & 137 & 0.01 & & 137 & & 0.02 \\
\hline 10 & TC 40 & 3 & [609-6.83] & 36,000 & {$[623-$} & $.77]$ & 36,000 & [621-14.92] & 36,000 & [609-3.81] & 36,0 & & [609-5. & & 36,000 \\
\hline 11 & & 4 & [552-4.49] & 36,000 & {$[551-$} & & 36,000 & [556-7.73] & 36,000 & [548-1.10] & 36,0 & & [548-4. & & 36,000 \\
\hline 12 & & 5 & [522-1.29] & 36,000 & {$[522-$} & & 36,000 & [522-3.46] & 36,000 & 522 & 207 & & [522-3. & & 36,000 \\
\hline 13 & TE 40 & 3 & {$[708-23.21]$} & 36,000 & [714- & $.73]$ & $\underline{36,000}$ & [716-25.61] & 36,000 & [713-20.62] & 36,0 & & {$[713-2($} & 75] & 36,000 \\
\hline 14 & & 4 & [628-17.46] & 36,000 & [635- & $.01]$ & 36,000 & [638-17.30] & 36,000 & [635-16.03] & 36,0 & & [659-19 & & 36,000 \\
\hline 15 & & 5 & [597-14.02] & 36,000 & [594- & $.26]$ & $\underline{36,000}$ & [595-13.81] & 36,000 & [597-12.67] & 36,0 & & [594-1] & & 36,000 \\
\hline 16 & TR 40 & 3 & 176 & 4357 & [176- & $.24]$ & $\underline{36,000}$ & [176-10.32] & 36,000 & 176 & 184 & & 176 & & 1665 \\
\hline 17 & & 4 & 149 & 95.88 & 149 & & 2781 & 149 & $\underline{3582}$ & 149 & 420 & & 149 & & 790 \\
\hline 18 & & 5 & 139 & 13.56 & 139 & & 13.56 & $\underline{139}$ & $\underline{79.66}$ & 139 & 31.4 & & 139 & & 41.77 \\
\hline Pr. id. & Pr. type & $p$ & BMTZ/E5BTE & & & BMT & E6BTEF & & $\underline{\text { BMTZ/E7BTI }}$ & & & BMT & E8BTEF & & \\
\hline & & & [BP-Gap\%] & Time (sec & onds) & [BP- & ap\%] & Time (seconds) & [BP-Gap\%] & Time (sec & $\overline{\mathrm{dds})}$ & [BP- & ap\%] & Tim & (seconds) \\
\hline 1 & TC 20 & 3 & 340 & 0.13 & & 340 & & 0.08 & 340 & 0.11 & & 340 & & 0.33 & \\
\hline 2 & & 4 & 318 & 0.08 & & 318 & & 0.02 & 318 & 0.03 & & 318 & & 0.16 & \\
\hline 3 & & 5 & $\underline{312}$ & $\underline{0.08}$ & & 312 & & 0.02 & 312 & 0.02 & & 312 & & 0.05 & \\
\hline 4 & TE 20 & 3 & 449 & 49.39 & & 449 & & 47.75 & 449 & 42.58 & & 449 & & 152 & \\
\hline 5 & & 4 & 385 & 253.24 & & 385 & & 59.53 & 385 & 53.11 & & 385 & & 250 & \\
\hline 6 & & 5 & 366 & 1752.91 & & 366 & & 343 & 366 & 172.91 & & 366 & & 356 & \\
\hline 7 & TR 20 & 3 & 168 & 0.06 & & 168 & & 0.09 & 168 & 0.03 & & 168 & & 0.06 & \\
\hline 8 & & 4 & 146 & 0.03 & & 146 & & 0.02 & 146 & 0.02 & & 146 & & 0.02 & \\
\hline 9 & & 5 & 137 & 0.02 & & 137 & & 0.02 & 137 & 0.00 & & 137 & & 0.00 & \\
\hline 10 & TC 40 & 3 & 609 & 17802 & & 609 & & 13112 & 609 & 5905.14 & & 609 & & 103 & 37.70 \\
\hline 11 & & 4 & [548-0.91] & 36,000 & & 548 & & 10420 & 548 & 3817.08 & & 548 & & 792 & 0.50 \\
\hline 12 & & 5 & 522 & 5581.59 & & 522 & & 3353 & 522 & 3125.38 & & 522 & & 467 & \\
\hline 13 & TE 40 & 3 & [712-14.52] & 36,000 & & {$[708$} & 14.68] & 36,000 & [712-16.21] & 36,000 & & {$[708$} & $-16.01]$ & 36,0 & \\
\hline 14 & & 4 & [627-14.28] & 36,000 & & {$[631$} & $14.84]$ & 36,000 & [628-14.20] & 36,000 & & {$[628$} & $-14.02]$ & 36,0 & \\
\hline 15 & & 5 & [594-12.50] & 36,000 & & {$[597$} & $12.23]$ & 36,000 & [594-11.43] & 36,000 & & {$[594$} & $-11.81]$ & 36,0 & \\
\hline 16 & TR 40 & 3 & 176 & 11.04 & & 176 & & 17.44 & 176 & 6.73 & & 176 & & 11.4 & \\
\hline 17 & & 4 & 149 & 28.20 & & 149 & & 22.86 & 149 & 16.80 & & 149 & & 29.6 & \\
\hline 18 & & 5 & 139 & 9.89 & & 139 & & 2.98 & 139 & 6.84 & & 139 & & 22.4 & \\
\hline
\end{tabular}

els without constraints (30) and (31), IMTZ/EiITEF for $\boldsymbol{i}=\mathbf{2 , 3 , 4}$ can solve only one instance (Problem 12) with the best solution time being 14,443 seconds.

Of the existing models, the models with constraints (12) and (13) except BMTZ/E5BTEF, i.e., BMTZ/EiBTEF for $\boldsymbol{i}=\mathbf{6 , 7 , 8}$, can solve all TC 40 instances. BMTZ/E5BTEF cannot solve Problem 11. Of these models, BMTZ/E7BTEF and BMTZ/E6BTEF produce the best and worst solution times of 4283 seconds and 8962 seconds, respectively. Of the models without constraints (12) and (13), BMTZ/E3BTEF can solve only one instance while the others cannot solve any instance.

Regarding TE 40 instances, none can be solved by any of the existing and new models within the allotted time. The average optimality gaps change from $15.17 \%$ (IMTZ/E3ITEF) to $18.33 \%$ (IMTZ/ITEF and IMTZ/E1ITEF) for the new models without constraints (30) and (31) and from 12.73\% (IMTZ/E5ITEF) to $14.61 \%$ (IMTZ/E6ITEF) for the new models with constraints (30) and (31). Rel-M and IMTZ/E7ITEF have average optimality gaps of
$13.11 \%$ and $12.87 \%$, respectively. The average optimality gaps change from 16.44\% (BMTZ/E3BTEF) to 20\% (BMTZ/E1BTEF) for the existing models without constraints (12) and (13) and from 13.77\% (BMTZ/E8ITEF) and 13.95\% (BMTZ/E6BTEF and BMTZ/ E7BTEF) with constraints (12) and (13).

The overall results show that the proposed models give better solution times than the corresponding existing ones given by Gouveia (1995). For the test instances with the same size and hop value, the worst and best solution times are obtained for TE instances and TR instances, respectively. The models with constraints (12) and (13) in the existing ones and with constraints (30) and (31) in the new ones have better solution times than the ones without them even though they only slightly improve the LP bounds.

To see how much Rel-M is better than other models with respect to solution times, the solution times for TE 20, TC 40, and TR 40 instances, for which the optimal solutions are obtained and the solution times make a difference, are selected for compar- 
Table 4

Solution times and integrality gaps for the new models. Please see the caption in Table 3.

\begin{tabular}{|c|c|c|c|c|c|c|c|c|c|c|c|c|}
\hline \multirow[t]{2}{*}{ Pr. id. } & \multirow[t]{2}{*}{ Pr. type } & \multirow[t]{2}{*}{$p$} & \multicolumn{2}{|l|}{ Rel-M } & \multicolumn{2}{|l|}{ IMTZ/ITEF } & \multicolumn{2}{|l|}{ IMTZ/E1ITEF } & \multicolumn{2}{|l|}{ IMTZ/E2ITEF } & \multicolumn{2}{|l|}{ IMTZ/E3ITEF } \\
\hline & & & [BP-Gap\%] & $\begin{array}{l}\text { Time } \\
\text { (seconds) }\end{array}$ & [BP-Gap\%] & $\begin{array}{l}\text { Time } \\
\text { (seconds) }\end{array}$ & [BP-Gap\%] & $\begin{array}{l}\text { Time } \\
\text { (seconds) }\end{array}$ & [BP-Gap\%] & $\begin{array}{l}\text { Time } \\
\text { (seconds) }\end{array}$ & [BP-Gap\%] & $\begin{array}{l}\text { Time } \\
\text { (seconds) }\end{array}$ \\
\hline 1 & \multirow[t]{3}{*}{ TC 20} & 3 & 340 & 0.15 & $\underline{340}$ & $\underline{1.09}$ & 340 & 0.80 & 340 & 0.38 & 340 & 0.34 \\
\hline 2 & & 4 & 318 & 0.11 & $\underline{318}$ & $\underline{0.20}$ & 318 & 0.11 & 318 & 0.09 & 318 & 0.11 \\
\hline 3 & & 5 & 312 & 0.04 & 312 & 0.08 & 312 & 0.06 & 312 & 0.07 & 312 & 0.03 \\
\hline 4 & \multirow[t]{3}{*}{ TE 20} & 3 & 449 & 139 & 449 & 1891 & $\underline{449}$ & $\underline{1357.94}$ & 449 & 1936 & 449 & 1204 \\
\hline 5 & & 4 & 385 & 149 & 385 & 832 & 385 & 580.02 & 385 & 1055 & $\underline{385}$ & 1062 \\
\hline 6 & & 5 & 366 & 62.6 & $\underline{366}$ & $\underline{593}$ & 366 & 445.41 & 366 & 327 & $\overline{366}$ & $\overline{450}$ \\
\hline 7 & \multirow[t]{3}{*}{ TR 20} & 3 & 168 & 0.06 & 168 & 0.08 & 168 & 0.08 & 168 & 0.14 & 168 & 0.06 \\
\hline 8 & & 4 & 146 & 0.05 & 146 & 0.05 & 146 & 0.05 & $\underline{146}$ & $\underline{0.08}$ & 146 & 0.03 \\
\hline 9 & & 5 & 137 & 0.01 & 137 & 0.02 & 137 & 0.02 & $\overline{137}$ & 0.05 & $\underline{137}$ & $\underline{0.06}$ \\
\hline 10 & \multirow[t]{3}{*}{ TC 40} & 3 & 609 & 196 & [609-7.46] & 36,000 & [609-7.40] & 36,000 & [609-6.47] & 36,000 & [609-2.98] & 36,000 \\
\hline 11 & & 4 & 548 & 721 & [551-4.95] & 36,000 & [551-4.93] & 36,000 & [548-3.87] & 36,000 & [551-3.84] & 36,000 \\
\hline 12 & & 5 & 522 & 1492 & [522-0.61] & 36,000 & [522-0.59] & 36,000 & 522 & 14443 & 522 & 23278 \\
\hline 13 & \multirow[t]{3}{*}{ TE 40} & 3 & [713-13.18] & 36,000 & [713-21.49] & 36,000 & [713-21.48] & 36,000 & [718-23.10] & $\underline{36,000}$ & [708-18.92] & 36,000 \\
\hline 14 & & 4 & [627-13.39] & 36,000 & [645-18.48] & $\underline{36,000}$ & [645-18.47] & 36,000 & [628-16.12] & 36,000 & [631-14.60] & 36,000 \\
\hline 15 & & 5 & [594-12.76] & 36,000 & [599-15.03] & 36,000 & [599-15.00] & 36,000 & [599-14.97] & 36,000 & [597-11.98] & 36,000 \\
\hline 16 & \multirow[t]{3}{*}{ TR 40} & 3 & 176 & 2.01 & $\underline{176}$ & 15837 & 176 & 15837 & 176 & 4049 & 176 & 3522 \\
\hline 17 & & 4 & 149 & 11.76 & $\overline{149}$ & 712 & 149 & 712 & 149 & 754 & 149 & 344 \\
\hline 18 & & 5 & 139 & 5.86 & 139 & 27.44 & 139 & 27.44 & 139 & 46.25 & 139 & 47.36 \\
\hline 19 & \multirow[t]{3}{*}{ TC 60} & 3 & [866-6.64] & 36,000 & & & & & & & & \\
\hline 20 & & 4 & [792-9.39] & 36,000 & & & & & & & & \\
\hline 21 & & 5 & [734-6.84] & 36,000 & & & & & & & & \\
\hline 22 & \multirow[t]{3}{*}{ TE 60} & 3 & [1569-26.25] & 36,000 & & & & & & & & \\
\hline 23 & & 4 & [1353-23.77] & 36,000 & & & & & & & & \\
\hline 24 & & 5 & {$[1258-21.25]$} & 36,000 & & & & & & & & \\
\hline 25 & \multirow[t]{5}{*}{ TR 60} & 3 & 274 & 1233 & & & & & & & & \\
\hline 26 & & 4 & 207 & 2895 & & & & & & & & \\
\hline \multirow[t]{3}{*}{27} & & 5 & 189 & 567 & & & & & & & & \\
\hline & & & IMTZ/E4ITEF & & IMTZ/E5ITEF & & IMTZ/E6ITEF & & IMTZ/E7ITEF & & IMTZ/E8ITEF & \\
\hline & & & [BP-Gap\%] & $\begin{array}{l}\text { Time } \\
\text { (seconds) }\end{array}$ & [BP-Gap\%] & $\begin{array}{l}\text { Time } \\
\text { (seconds) }\end{array}$ & [BP-Gap\%] & $\begin{array}{l}\text { Time } \\
\text { (seconds) }\end{array}$ & [BP-Gap\%] & $\begin{array}{l}\text { Time } \\
\text { (seconds) }\end{array}$ & [BP-Gap\%] & $\begin{array}{l}\text { Time } \\
\text { (seconds) }\end{array}$ \\
\hline 1 & TC 20 & 3 & 340 & 0.45 & 340 & 0.13 & 340 & 0.09 & 340 & 0.14 & 340 & 0.14 \\
\hline 2 & & 4 & 318 & 0.11 & 318 & 0.05 & 318 & 0.05 & 318 & 0.06 & 318 & 0.06 \\
\hline 3 & & 5 & $\underline{312}$ & $\underline{0.08}$ & 312 & 0.05 & 312 & 0.03 & 312 & 0.03 & 312 & 0.06 \\
\hline 4 & TE 20 & 3 & 449 & 2550 & 449 & 37.03 & 449 & 22.74 & 449 & 30.16 & 449 & 81.34 \\
\hline 5 & & 4 & 385 & 301 & 385 & 215.81 & 385 & 128.03 & 385 & 90.38 & 385 & 229 \\
\hline 6 & & 5 & 366 & 448 & 366 & 129.86 & 366 & 108.13 & 366 & 104.13 & 366 & 332 \\
\hline 7 & TR 20 & 3 & $\underline{168}$ & $\underline{0.16}$ & 168 & 0.02 & 168 & 0.03 & 168 & 0.03 & 168 & 0.03 \\
\hline 8 & & 4 & $\overline{146}$ & $\overline{0.03}$ & 146 & 0.02 & 146 & 0.02 & 146 & 0.02 & 146 & 0.01 \\
\hline 9 & & 5 & 137 & 0.02 & 137 & 0.02 & 137 & 0.02 & 137 & 0.02 & 137 & 0.02 \\
\hline 10 & TC 40 & 3 & [611-6.32] & 36,000 & 609 & 614.34 & 609 & 3533 & 609 & 9070 & 609 & 6028 \\
\hline 11 & & 4 & [548-3.76] & 36,000 & 548 & 6043 & 548 & 1884 & 548 & 17954 & 548 & 10411 \\
\hline 12 & & 5 & 522 & 17725 & 522 & 10693 & 522 & 5266 & 522 & 4150 & 522 & 4555 \\
\hline 13 & TE 40 & 3 & [725-21.68] & 36,000 & [708-11.89] & 36,000 & [708-16.08] & 36,000 & [712-13.20] & 36,000 & [708-14.60] & 36,000 \\
\hline 14 & & 4 & [628-16.11] & 36,000 & [627-14.14] & 36,000 & [627-15.05] & 36,000 & [627-13.46] & 36,000 & [627-14.09] & 36,000 \\
\hline 15 & & 5 & [594-11.65] & 36,000 & [594-12.16] & 36,000 & [597-12.70] & 36,000 & [599-11.94] & 36,000 & [599-11.97] & 36,000 \\
\hline 16 & TR 40 & 3 & 176 & 1638 & 176 & 2.67 & 176 & 32.88 & 176 & 28.42 & 176 & 23.91 \\
\hline 17 & & 4 & 149 & $\underline{857}$ & 149 & 33.05 & 149 & 43.95 & 149 & 39.00 & 149 & 64.70 \\
\hline 18 & & 5 & $\overline{139}$ & $\overline{39.23}$ & 139 & 13.42 & 139 & 19.56 & 139 & 33.33 & 139 & $\underline{56.08}$ \\
\hline
\end{tabular}

ison. Of the models proposed by Gouveia (1995), BMTZ/E7BTEF gives the best results with average solution times of 89.53 , 4282.53, and 10.12 seconds for TE 20, TC 40, and TR 40 instances, respectively. The average solution time for all 9 instances is 1460.73 seconds. Of the new models, Rel-M gives the best results with average solution times of $117.15,803.53$, and 6.54 seconds for TE 20, TC 40, and TR 40 instances, respectively. The average solution time for all 9 instances is 309.07 seconds. IMTZ/E6ITEF gives the second best results with solution times of $86.3,3561.5$, and 32.13 seconds for TE 20, TC 40, and TR 40 instances, respectively.
The average solution time for all 9 instances is 1226.63 seconds. Clearly, Rel-M gives significantly better solution times on the average than all other models.

For further evaluation of Rel-M, TC 60, TE 60, and TR 60 instances are also solved. The results in Table 4 show that all TR 60 instances can be solved to optimality with solution times changing from 567 seconds (Problem 27) to 2895 seconds (Problem 26). None of the TC 60 and TE 60 instances can be solved to optimality. The optimality gaps change from 6.64\% (Problem 19) 
to $9.39 \%$ (Problem 20) for TC instances and from 21.25\% (Problem 24) to $26.25 \%$ (Problem 22) for TE instances.

Recall that we use constraints (32) in Rel-M instead of constraints (21). When we solve Problems 10, 11, and 12 with constraints (21) instead of constraints (32), we get solution times of 694,8596 , and 10,330 seconds, respectively. Rel-M with constraints (32) gives the solution times of 196,721 , and 1492 seconds for the same problems (Table 4), respectively. This indicates that the use of constraints (32) in Rel-M instead of constraints (21) cuts down the solution times by about three to twelve times in the given instances.

\section{Tests for different hop values and network sizes}

To see the effect of hop values on the LP bounds, objective function values, and solution times, the models with the best solution times, Rel-M, IMTZ/E6ITEF, and BMTZ/E7BTEF, are solved for different hop values changing from 4 to 40 by using the TE 40 instance. The results are given in Table 5 .

The results show that the LP bounds and the optimal objective function values stabilize after $p=8$ and $p=18$, respectively, for all three models. IMTZ/E6ITEF has better LP bounds than the other two models for all values of $p$. Although BMTZ/E7BTEF has better LP bounds than Rel-M for $p=4$ and $p=6$, they have the same LP bounds for $p \geqslant 8$. The optimal solutions cannot be obtained until a certain value of $p$ in the allotted time. However, the optimal solutions are obtained in a very short time after that certain value of $p$. Specifically, Rel-M can find optimal solutions for $p \geqslant 14$ while IMTZ/E6ITEF and BMTZ/E7BTEF can find optimal solutions for $p \geqslant 16$. Fig. 3 shows how the LP bounds and optimal objective function values change depending on the hop values. The solution times of Rel-M, IMTZ/E6ITEF, and BMTZ/E7BTEF for $p \geqslant 16$ change from 49 to 164 seconds with an average of 101 seconds, from 123 to 575 seconds with an average of 235 seconds, and from 58 to 307 seconds with an average of 182 seconds, respectively.

To see how the models behave depending on the number of nodes in the network, TC instances with the number of nodes changing from 20 to 40 with $p=4$ are created and solved by using Rel-M, IMTZ/E6ITEF, and BMTZ/E7BTEF. The results are given in Table 6 . The results show that the models behave similarly in the sense that the correlation coefficients for the LP bounds and solution times of the models are almost 1 for all pairs of models as shown in Fig. 4. That is, if a problem is hard (easy) to solve for a

Table 5

LP bounds, solution times, and integrality gaps for TE 40 instance with different hop values. Please see the caption in Table 3 .

\begin{tabular}{|c|c|c|c|c|c|c|c|c|c|c|c|}
\hline \multirow[t]{2}{*}{ Pr. id. } & \multirow[t]{2}{*}{ Pr. type } & \multirow[t]{2}{*}{$p$} & \multicolumn{3}{|l|}{ Rel-M } & \multicolumn{3}{|c|}{ IMTZ/E6ITEF } & \multicolumn{3}{|c|}{ BMTZ/E7BTEF } \\
\hline & & & LP bound & [BP-Gap\%] & Time (seconds) & LP bound & [BP-Gap\%] & Time (seconds) & LP bound & [BP-Gap\%] & Time (seconds) \\
\hline 1 & TE 40 & 4 & 481.06 & [627-13.39] & 36,000 & 487.69 & [627-15.05] & 36,000 & 481.78 & [628-14.2] & 36,000 \\
\hline 2 & & 6 & 480.17 & [569-10.64] & 36,000 & 486.33 & [568-10.36] & 36,000 & 480.36 & [568-10.4] & 36,000 \\
\hline 3 & & 8 & 480.00 & [537-6.83] & 36,000 & 486.00 & [542-7.66] & 36,000 & 480.00 & [537-6.35] & 36,000 \\
\hline 4 & & 10 & 480.00 & [520-3.81] & 36,000 & 486.00 & [520-4.10] & 36,000 & 480.00 & [520-3.55] & 36,000 \\
\hline 5 & & 12 & 480.00 & [514-2.53] & 36,000 & 486.00 & [514-2.83] & 36,000 & 480.00 & [514-2.89] & 36,000 \\
\hline 6 & & 14 & 480.00 & 506 & 12756 & 486.00 & [506-1.33] & 36,000 & 480.00 & [506-0.64] & 36,000 \\
\hline 7 & & 16 & 480.00 & 498 & 164.18 & 486.00 & 498 & 349.97 & 480.00 & 498 & 307.29 \\
\hline 8 & & 18 & 480.00 & 496 & 51.37 & 486.00 & 496 & 123.33 & 480.00 & 496 & 96.78 \\
\hline 9 & & 20 & 480.00 & 496 & 48.75 & 486.00 & 496 & 134.02 & 480.00 & 496 & 178.86 \\
\hline 10 & & 22 & 480.00 & 496 & 71.35 & 486.00 & 496 & 128.68 & 480.00 & 496 & 135.82 \\
\hline 11 & & 24 & 480.00 & 496 & 158.45 & 486.00 & 496 & 253.78 & 480.00 & 496 & 286.48 \\
\hline 12 & & 26 & 480.00 & 496 & 95.82 & 486.00 & 496 & 204.67 & 480.00 & 496 & 188.61 \\
\hline 13 & & 28 & 480.00 & 496 & 53.82 & 486.00 & 496 & 248.28 & 480.00 & 496 & 83.16 \\
\hline 14 & & 30 & 480.00 & 496 & 98.51 & 486.00 & 496 & 168.53 & 480.00 & 496 & 221.37 \\
\hline 15 & & 32 & 480.00 & 496 & 142.76 & 486.00 & 496 & 173.41 & 480.00 & 496 & 239.26 \\
\hline 16 & & 34 & 480.00 & 496 & 101.82 & 486.00 & 496 & 226.25 & 480.00 & 496 & 208.53 \\
\hline 17 & & 36 & 480.00 & 496 & 120.46 & 486.00 & 496 & 219.92 & 480.00 & 496 & 303.19 \\
\hline 18 & & 38 & 480.00 & 496 & 136.80 & 486.00 & 496 & 253.53 & 480.00 & 496 & 58.41 \\
\hline 19 & & 40 & 480.00 & 496 & 74.83 & 486.00 & 496 & 575.21 & 480.00 & 496 & 61.81 \\
\hline
\end{tabular}

¿Rel-M IIMTZ/EGITEF $\square B M T Z / E 7 B T E F$

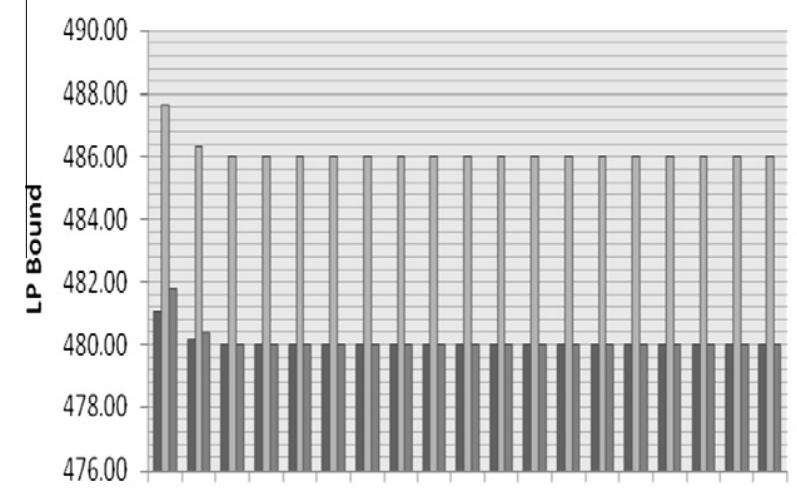

46810121416182022242628303234363840

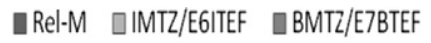

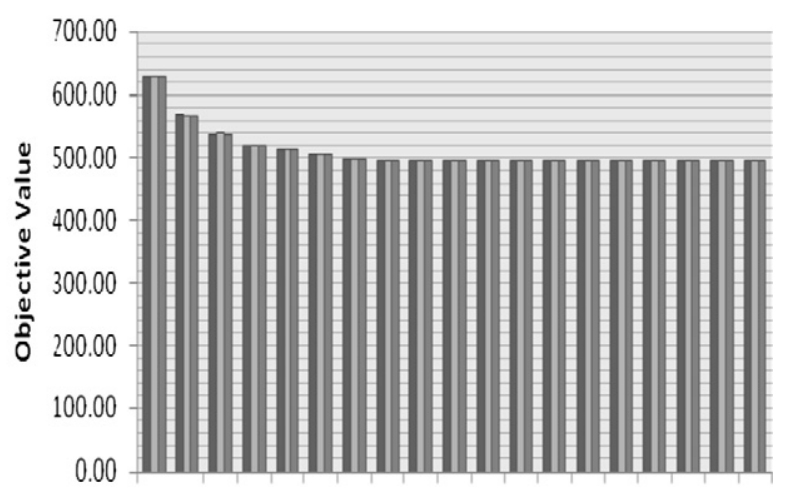

46810121416182022242628303234363840

p

$p$

Fig. 3. The effect of hop values on the LP bounds and optimal objective function values. 
Table 6

LP bounds, solution times, and integrality gaps for variable network sizes with $p=4$. Please see the caption in Table 3 .

\begin{tabular}{|c|c|c|c|c|c|c|c|c|c|c|c|}
\hline \multirow[t]{2}{*}{ Pr. id. } & \multirow[t]{2}{*}{ Pr. type } & \multirow[t]{2}{*}{$|n|$} & \multicolumn{3}{|l|}{ Rel-M } & \multicolumn{3}{|c|}{ IMTZ/E6ITEF } & \multicolumn{3}{|c|}{ BMTZ/E7BTEF } \\
\hline & & & LP bound & [BP-Gap\%] & Time (seconds) & LP bound & [BP-Gap\%] & Time (seconds) & LP bound & [BP-Gap\%] & Time (seconds) \\
\hline 1 & $\mathrm{TC}$ & 20 & 304.36 & 359 & 3.28 & 304.70 & 359 & 10.79 & 304.67 & 359 & 4.85 \\
\hline 2 & & 22 & 317.18 & 334 & 0.12 & 317.29 & 334 & 0.30 & 317.27 & 334 & 0.22 \\
\hline 3 & & 24 & 300.00 & 344 & 6.33 & 303.00 & 344 & 8.60 & 299.80 & 344 & 7.04 \\
\hline 4 & & 26 & 338.75 & 386 & 0.53 & 339.33 & 386 & 2.39 & 338.37 & 386 & 1.72 \\
\hline 5 & & 28 & 340.00 & 397 & 1.20 & 340.00 & 397 & 3.01 & 340.00 & 397 & 1.04 \\
\hline 6 & & 30 & 349.75 & 408 & 18.10 & 350.27 & 408 & 63.49 & 350.40 & 408 & 15.18 \\
\hline 7 & & 32 & 341.92 & 432 & 2786 & 342.11 & 432 & 4352.00 & 338.32 & 432 & 2034.00 \\
\hline 8 & & 34 & 350.74 & 385 & 28.05 & 351.95 & 385 & 86.03 & 350.27 & 385 & 22.39 \\
\hline 9 & & 36 & 377.00 & 450 & 77.53 & 378.21 & 450 & 751.00 & 377.75 & 450 & 188.15 \\
\hline 10 & & 38 & 386.94 & [475-3.72] & 36,000 & 387.54 & [475-4.91] & 36,000 & 384.69 & [475-3.26] & 36,000 \\
\hline 11 & & 40 & 450.25 & 526 & 2712 & 450.33 & 526 & 12692 & 450.40 & 526 & 6281 \\
\hline
\end{tabular}

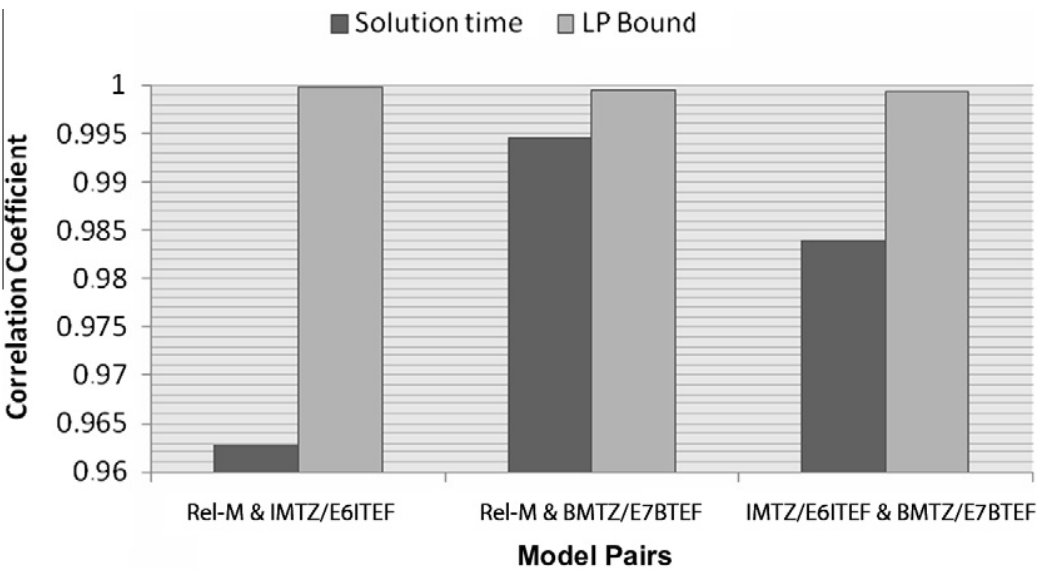

Fig. 4. The relationship of the models with respect to LP bounds and solution times.

model, that model is also hard (easy) to solve for other models. A similar result is observed for the hop values (Table 5).

\section{Conclusion}

The computational results that we get in this paper indicate that the new models we propose in the paper give considerably better bounds and solution times than their counterparts in the literature and that the new sets of constraints that we propose to improve the existing formulations are competitive with earlier proposed liftings to Miller-Tucker-Zemlin constraints in Gouveia (1995), some of which are based on the well-known, strong liftings of Desrochers and Laporte (1991). Our computational tests indicate that the best solution times result from the new formulations that do not include the earlier liftings offered in Gouveia (1995). The use of these liftings in conjunction with the set of constraints proposed in this paper tends to make the solution times worse.

\section{References}

Achuthan, N.R., Caccetta, L., Caccetta, P.A. Geelen, J.F., 1994. Computational methods for the diameter restricted minimum weight spanning tree problem. Australasian Journal of Combinatorics 10, 51-71.

Akgun, I., Tansel, B.C., 2009. Degree constrained minimum spanning tree problem: New formulation via Miller-Tucker-Zemlin constraints. Research Report, Bilkent University, Department of Industrial Engineering, Bilkent, Ankara (under review).

Akgun, I., Tansel, B.C., 2010. Min-degree constrained minimum spanning tree problem: New formulation via Miller-Tucker-Zemlin constraints. Computers and Operations Research 37 (1), 72-82.

Ahuja, R., Magnanti, T., Orlin, J., 1993. Network Flows: Theory, Algorithms and Applications. Prentice Hall.
Alfandari, L., Paschos, V.Th., 1999. Approximating minimum spanning tree of depth 2. International Transactions in Operational Research 6, 607-622.

Applegate, D.L., Bixby, R.E., Chvatal, V., Cook, W.J., 2007. The Traveling Salesman Problem: A Computational Study. Princeton Series in Applied Mathematics. Princeton University Press.

Balakrishnan, A., Altinkemer, K., 1992. Using a hop-constrained model to generate alternative communication network design. ORSA Journal on Computing 4 192-205.

Dahl, G., 1998. The 2-hop spanning tree problem. Operations Research Letters 23, 21-26.

Dahl, G., Flatbert, T., Foldnes, N., Gouveia, L., 2004. The jump formulation for the hop-constrained minimum spanning tree problem. Technical Report, Centro de Investigaciao Operacional, Faculdade de Ciencias da Universidade de Lisboa.

Dahl, G., Gouveia, L., Requeijo, C., 2006. On formulations and methods for the hopconstrained minimum spanning tree problem. In: Resende, M.G.C., Pardalos, P.M., Pardalos, P.M. (Eds.), Handbooks of Telecommunications. Springer, pp. 493-515.

Desrochers, M., Laporte, G., 1991. Improvements and extensions to the MillerTucker-Zemlin subtour elimination constraints. Operations Research Letters 10, 27-36.

Gavish, B., 1985. Augmented Lagrangian based algorithms for centralized network design. IEEE Transactions on Communications 33, 1247-1257.

Gouveia, L., 1995. Using the Miller-Tucker-Zemlin constraints to formulate minimal spanning trees with hop constraints. Computers and Operations Research 22, 959-970.

Gouveia, L., 1996. Multicommodity flow models for spanning trees with hop constraints. European Journal of Operational Research 95, 178-190.

Gouveia, L., 1998. Using variable redefinition for computing lower bounds for minimum spanning and Steiner trees with hop constraints. INFORMS Journal on Computing 10, 180-188.

Gouveia, L., Magnanti, T.L., 2003. Network flow models for designing diameterconstrained minimum-spanning and Steiner trees. Networks 41 (3), 159-173.

Gouveia, L., Patricio, P., Sousa, A., Valadas, R., 2003. MPLS over WDM network design with packet level QoS constraints based on ILP models. Proceedings of INFOCOM 2003, 22nd Annual Joint Conference of the IEEE Computer and Communications, vol. 1. IEEE Societies, pp. 576-586.

Gouveia, L., Pires, J.M., 1999. The asymmetric travelling salesman problem and a reformulation of the Miller-Tucker-Zemlin constraints. European Journal of Operational Research 112, 134-146. 
Gouveia, L., Requejo, C., 2001. A new Lagrangian relaxation approach for the hopconstrained minimum spanning tree problem. European Journal of Operational Research 132 (3), 539-552.

Gouveia, L., Paiasa, A., Sharmab, D., 2008. Modeling and solving the rooted distanceconstrained minimum spanning tree problem. Computers and Operations Research 35, 600-613.

Gouveia, L., Simonetti, L., Uchoa, E., 2010. Modeling hop-constrained and diameterconstrained minimum spanning tree problems as Steiner tree problems over layered graphs. Mathematical Programming. <http://dx.doi.org/10.1007/ s10107-009-0297-2>.

Gruber, M., Raidl, G.R., 2005. A new 0-1 ILP approach for the bounded diameter minimum spanning tree problem. In: Proceedings of the Second International Network Optimization Conference, Lisbon, pp. 178-185.

Gutin, G., Punnen, A.P. (Eds.), 2002. The Traveling Salesman Problem and Its Variations. Combinatorial Optimization, vol. 12. Kluwer, Dordrecht.

Hwang, F.K., Richards, D.S., Winter, P., 1992. The Steiner Tree Problems. Annals of Discrete Mathematics. North-Holland.

ILOG/CPLEX 9.0, 2003. Reference Manual.

Kerivin, H., Mahjoub, A.R., 2005. Design of survivable networks: A survey. Networks 46 (1), 1-21.

Kruskal, J.B., 1956. On the shortest spanning subtree of a graph and the traveling salesman problem. Proceedings of the American Mathematics Society 7 (1), 48 50.

LeBlanc, L., Chifflet, J., Mahey, P., 1999. Packet routing in telecommunication networks with path and flow restrictions. INFORMS Journal on Computing 11, 188-197.

Maculan, N., 1987. The Steiner problem in graphs. Annals of Discrete Mathematics $31,185-212$.
Magnanti, T., Wolsey, L., 1995. Optimal trees. In: Ball, M.O., Magnanti, T.L., Monma, C.L., Nemhauser, G.L. (Eds.), Network Models. Handbooks in Operations Research and Management Science, vol. 7. North-Holland, Amsterdam, pp. 503-615.

Manyem, P., Stallmann, M., 1996. Some approximation results in multicasting Technical Report, North Carolina State University.

Miller, C., Tucker, A., Zemlin, R., 1960. Integer programming formulation of traveling salesman problems. Journal of the ACM 7, 326-329.

Nemhauser, G.L., Wolsey, L.A., 1988. Integer and Combinatorial Optimization. John Wiley \& Sons.

Orman, A.J., Williams, H.P., 1999. A Survey of Different Integer Programming Formulations of the Traveling Salesman Problem. Preprint Series \# OR101. Faculty of Mathematical Studies, University of Southampton, Southampton, England.

Pirkul, H., Soni, S., 2003. New formulations and solution procedures for the hop constrained network design problem. European Journal of Operational Research $148,126-140$.

Prim, R., 1957. Shortest connection networks and some generalizations. Bell System Technical Journal 36, 1389-1401.

Santos, A.C., Lucena, A., Ribeiro, C.C., 2004. Solving diameter constrained minimum spanning tree problems in dense graphs. Experimental and Efficient Algorithms. Lecture Notes in Computer Science, vol. 3059. Springer, Berlin, pp. 458-467.

Sherali, H.D., Driscoll, P.J., 2002. On tightening the relaxations of Miller-TuckerZemlin formulations for asymmetric traveling salesman problems. Operations Research 50 (4), 656-669.

Woolston, K Albin, S, 1988. The design of centralized networks with reliability and availability constraints. Computers and Operations Research 15, 207-217. 